\title{
A partial form of recessive STAT1 deficiency in humans
}

\author{
Ariane Chapgier,,$^{1,2}$ Xiao-Fei Kong, 1,2,3,4 Stéphanie Boisson-Dupuis, 1,2,4

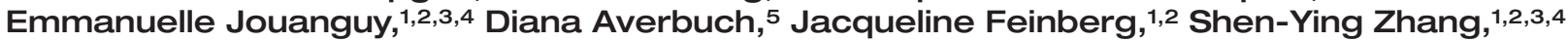 \\ Jacinta Bustamante,1,2 Guillaume Vogt,1,2 Julien Lejeune,1,2 Eleonore Mayola,1,2 \\ Ludovic de Beaucoudrey,1,2 Laurent Abel,1,2,4 Dan Engelhard,5 and Jean-Laurent Casanova1,2,3,4,6
}

\begin{abstract}
1 Laboratory of Human Genetics of Infectious Diseases, Necker Faculty, INSERM U550, Paris, France. ${ }^{2}$ Necker Medical School, University Paris Descartes, Paris, France. ${ }^{3}$ French-Chinese Laboratory of Genetics and Life Sciences, Ruijin Hospital, Shanghai Jiaotong University, Shanghai, People's Republic of China. “Laboratory of Human Genetics of Infectious Diseases, Rockefeller Branch, The Rockefeller University, New York, New York, USA. '5epartment of Pediatrics, Department of Infectious Diseases, and AIDS Center, Hadassah University, Hospital Ein Karem, Jerusalem, Israel. ${ }^{6}$ Pediatric Immunology and Hematology Unit, Necker Enfants Malades Hospital, Paris, France.
\end{abstract}

\begin{abstract}
Complete STAT1 deficiency is an autosomal recessive primary immunodeficiency caused by null mutations that abolish STAT1-dependent cellular responses to both IFN- $\alpha / \beta$ and IFN- $\gamma$. Affected children suffer from lethal intracellular bacterial and viral diseases. Here we report a recessive form of partial STAT1 deficiency, characterized by impaired but not abolished IFN $-\alpha / \beta$ and IFN $-\gamma$ signaling. Two affected siblings suffered from severe but curable intracellular bacterial and viral diseases. Both were homozygous for a missense STAT1 mutation: g.C2086T (P696S). This STAT1 allele impaired the splicing of STAT1 mRNA, probably by disrupting an exonic splice enhancer. The misspliced forms were not translated into a mature protein. The allele was hypofunctional, because residual full-length mRNA production resulted in low but detectable levels of normally functional STAT1 proteins. The P696S amino acid substitution was not detrimental. The patients' cells, therefore, displayed impaired but not abolished responses to both IFN- $\alpha$ and IFN- $\gamma$. We also show that recessive STAT1 deficiencies impaired the IL-27 and IFN- $\lambda 1$ signaling pathways, possibly contributing to the predisposition to bacterial and viral infections, respectively. Partial recessive STAT1 deficiency is what we believe to be a novel primary immunodeficiency, resulting in impairment of the response to at least 4 cytokines (IFN- $\alpha / \beta$, IFN- $\gamma$, IFN- $\lambda 1$, and IL-27). It should be considered in patients with unexplained, severe, but curable intracellular bacterial and viral infections.
\end{abstract}

\section{Introduction}

STAT1 is a key signaling component of IFN responses. There are 2 STAT1 mRNAs, STAT1A and STAT1B, which use distinct polyadenylation sites. STAT1A is transcribed and spliced from 25 exons, whereas $S T A T 1 B$ is transcribed and spliced from the first 23 exons, ending downstream from the STAT1A exon 23 splice site. The 2 resulting proteins thus have different carboxyterminal ends, as STAT1 $\beta$ lacks the transactivator domain (Figure 1A) (1, 2 ). Bioactive STAT $1 \alpha$ is the predominantly expressed form, and the transcriptionally inactive STAT $1 \beta$ modulates the effects of STAT $1 \alpha(3,4)$. Following IFN- $\alpha$ stimulation, the heterodimeric IFN- $\alpha$ receptor is phosphorylated by the associated Janus kinases JAK1 and TYK2, creating a docking site for a single STAT2 molecule, which is in turn phosphorylated. One latent STAT1 molecule is recruited by phosphorylated STAT2 (P-STAT2) and phosphorylated on its Tyr701 residue (1). Active phosphorylated STAT1/STAT2 heterodimers are released into the cytosol, in which they combine with IFN-stimulated gene factor $3 \gamma$ (ISGF3- $\gamma$ ), also

Authorship note: Xiao-Fei Kong, Stéphanie Boisson-Dupuis, and Emmanuelle Jouanguy contributed equally to this work.

Conflict of interest: The authors have declared that no conflict of interest exists.

Nonstandard abbreviations used: BCG, bacille Calmette-Guérin; EBV-B cell, EBVtransformed B cell; ESE, exonic splice enhancer; ESS, exonic splice silencer; GAF, gamma-activating factor; GAS, gamma-activating sequence; HSV, herpes simplex virus; ISGF3, IFN-stimulated gene factor 3; ISRE, IFN- $\alpha$ sequence response element; MSMD, Mendelian predisposition to mycobacterial diseases; P-STAT, phosphorylated STAT; P-Tyr, phosphorylated Tyr; VSV, vesicular stomatitis virus.

Citation for this article: J. Clin. Invest. doi:10.1172/JCI37083 known as p48 or IRF9, to form ISGF3. ISGF3 is translocated to the nucleus (5), in which it binds to IFN- $\alpha$ sequence response elements (ISREs) in the promoters of target genes via the DNA-binding domains of STAT1 and ISGF3- $\gamma$ and activates transcription through the transactivator domain of STAT2 (1). IFN- $\gamma$ stimulation leads to activation of the associated Janus kinases JAK1 and JAK2, which phosphorylate the tetrameric IFN- $\gamma$ receptor, creating a docking site for 2 latent STAT1 molecules. These molecules are phosphorylated on their Tyr701 residues and are released into the cytosol as phosphorylated active STAT1 homodimers, forming gamma-activating factor $(\mathrm{GAF})(1,6,7)$. GAF is translocated to the nucleus (8), where it binds to gamma-activating sequences (GASs) in the promoters of target genes and activates transcription through the transactivator domain of STAT1 (1). The role of STAT1 in protective immunity to diverse viruses (9-12), bacteria $(9,13-15)$, and parasites $(16,17)$ in vivo has been documented in mouse models of experimental infection.

Two types of partial STAT1 deficiency, both showing dominant inheritance, have been described in human patients with Mendelian predisposition to mycobacterial diseases (MSMD). MSMD is characterized by the occurrence of clinical disease caused by weakly virulent mycobacteria in otherwise healthy patients (18, 19). Other infections are rare, with the exception of salmonellosis, which is found in less than half the patients. The heterozygous L706S STAT1 mutation in the tail segment domain of STAT1 (Figure 1A) impairs Tyr701 phosphorylation, preventing the formation of ISGF3 and GAF complexes following stimulation with IFN- $\alpha$ and IFN- $\gamma$, respectively $(18,19)$. Heterozygous STAT1 
A

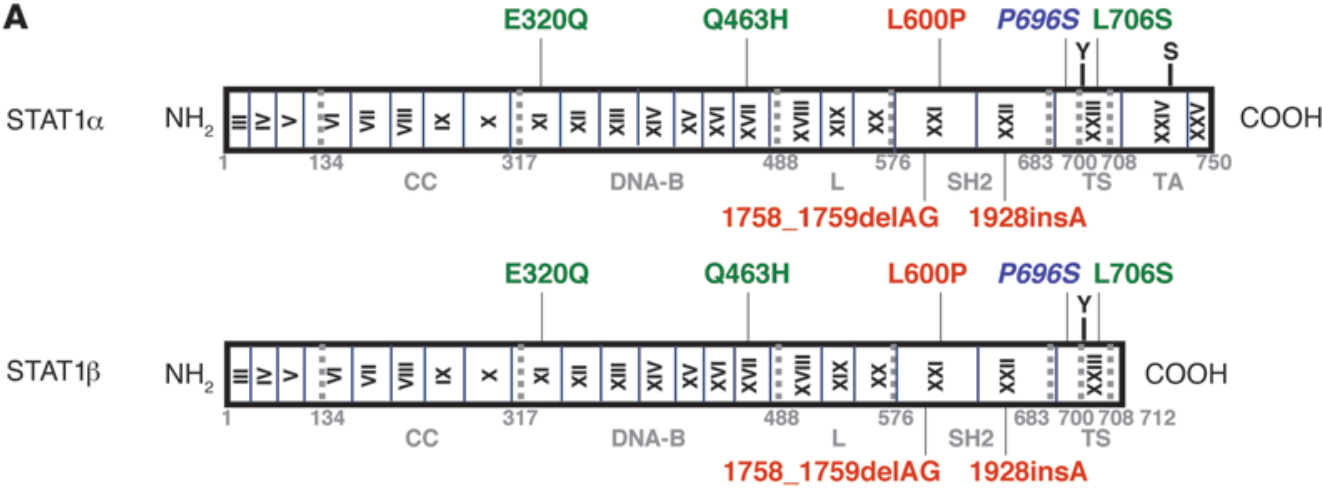

B

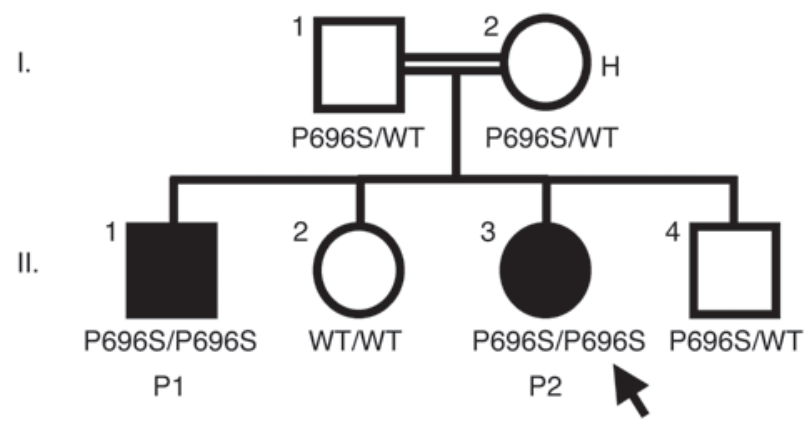

C

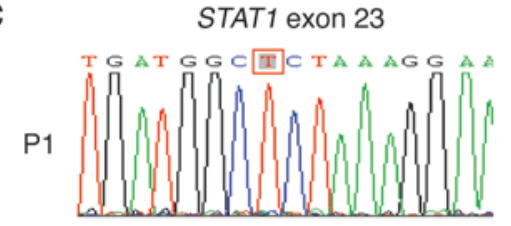

P696S/

P696S

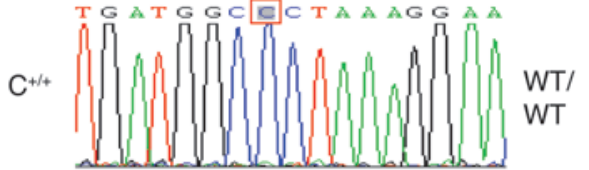

\section{Figure 1}

STAT1 P696S is associated with salmonellosis and viral disease. (A) Human STAT1 $\alpha$ and STAT1 $\beta$ isoforms are shown, with their known pathogenic mutations. Coding exons are numbered with Roman numerals and delimited by a vertical bar. Regions corresponding to the coiled-coil domain (CC), DNA-binding domain (DNA-B), linker domain (L), SH2 domain (SH2), tail segment domain (TS), and transactivator domain (TA) are indicated in gray, together with their amino acid boundaries, and are delimited by gray dotted lines. Tyr701 (Y) and Ser727 (S) are indicated. Mutations in red are recessive and associated with complete STAT1 deficiency in homozygous individuals. Mutations in green are associated with partial STAT1 deficiency in heterozygous individuals. The mutation in blue is a partial recessive mutation associated with partial STAT1 deficiency in homozygous individuals. The mutation reported here for what we believe to be the first time is indicated in italics. (B) STAT1 genotype and clinical phenotype of the kindred. Members II.1 and II.3 presented salmonellosis, and II.3 also presented viral diseases. These 2 individuals are subsequently referred to as P1 and P2, respectively. Individuals with clinical disease are indicated in black, and healthy individuals are shown in white. The mother, who is heterozygous for the STAT1 P696S mutation, is subsequently referred to as H. STAT1 genotypes are indicated under each individual. The index case is indicated with an arrow. (C) Genomic sequences in the sense orientation of exon 23 of STAT1 in a healthy control and in $\mathrm{P} 1 . \mathrm{C}^{+/+}$indicates a healthy control.

mutations E320Q and Q463H in the DNA-binding domain of STAT1 (Figure 1A) impair ISGF3 and GAF binding to ISREs and GAS elements upon IFN- $\alpha$ and IFN- $\gamma$ stimulation, respectively (19). Transfection experiments with STAT1-deficient cells have shown that these alleles are deleterious for both IFN- $\gamma$ and IFN- $\alpha$ responses. However, although they impair IFN- $\gamma$-induced, GAFmediated immunity in heterozygous patients, IFN- $\alpha$-induced, ISGF3-mediated immunity is maintained, accounting for the autosomal dominant MSMD observed in patients. By contrast, a recessive form of complete STAT1 deficiency has been described in other patients, with a related but different syndrome of susceptibility to both mycobacterial and viral diseases $(20,21)$. These patients are homozygous for the 1758_1759delAG, L600P (20), or 1928insA (21) STAT1 mutations (Figure 1A). These alleles are null, as they are associated with a complete absence of STAT1 production and function in terms of both the IFN- $\alpha$-induced, ISGF3dependent and the IFN- $\gamma$-induced, GAF-dependent responses. The abolition of responses to IFN- $\alpha / \beta$ and IFN $-\gamma$ accounts for the predisposition to life-threatening illnesses caused by viruses and mycobacteria, respectively. We describe here what we believe to be a novel form of autosomal recessive STAT1 deficiency, with a hypomorphic mutation conferring partial, as opposed to complete, STAT1 deficiency in 2 siblings suffering from severe but curable intracellular bacterial and viral diseases.

\section{Results}

Identification of the P696S STAT1 allele in 2 siblings. We investigated 2 siblings (herein called patient 1 [P1] and P2) born to consanguineous parents, originating from and living in Israel (Figure $1 \mathrm{~B}$ and see Methods). Neither had been vaccinated with bacille CalmetteGuérin (BCG). P1 and P2 developed recurrent and disseminated Salmonella infections, and $\mathrm{P} 2$ also suffered from recurrent herpes virus infections. An association of herpes virus and intracellular bacterial infections has been documented in patients with complete STAT1 deficiency with impaired IFN- $\alpha / \beta$ - and IFN- $\gamma$-mediated immunity (20). A patient with TYK2 deficiency presented a broader phenotype, including staphylococcal disease and high serum IgE levels - not found in our patients - due to impaired cellular responses to various cytokines, including IFN- $\alpha / \beta$, IL- 6 , IL-10, IL-23, and IL-12 (22). We therefore began by sequencing the STAT1 exons and flank- 

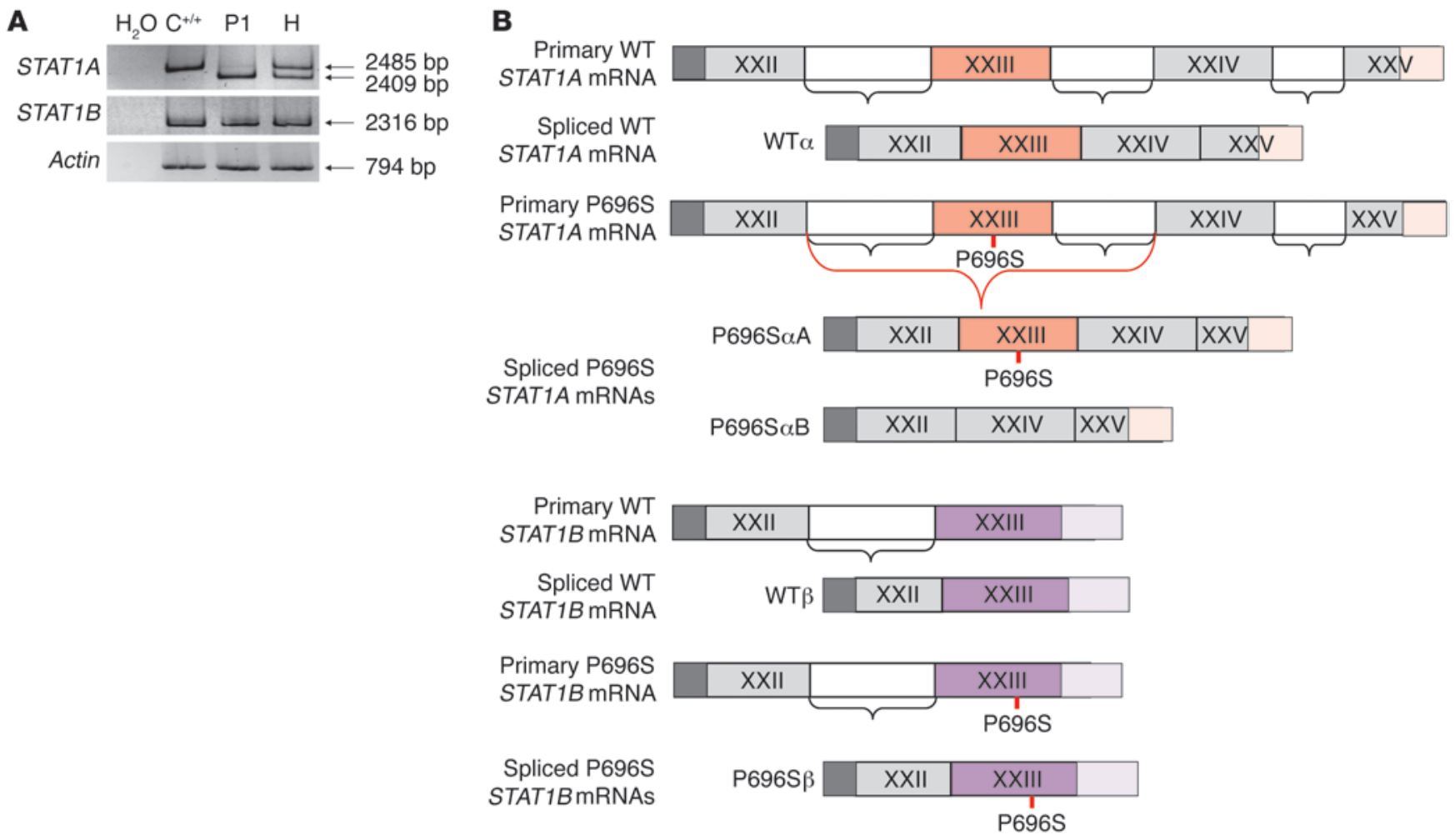

\section{Figure 2}

STAT1 P696S is associated with a predominant splicing defect. (A) PCR of full-length STAT1A, STAT1B, and ACTIN amplified from cDNA from $\mathrm{C}^{+/+}, \mathrm{P} 1$ (homozygous for the P696S STAT1 mutation), and $\mathrm{H}$. The sizes of the amplicons are indicated. This result is representative of 5 independent experiments. (B) Schematic representation of the splicing events identified in STAT1A and STAT1B WT or P696S mutant. The ends of the WT and P696S STAT1 mRNAs are shown, with their corresponding spliced forms. The exons are numbered with Roman numerals and represented in gray boxes, with the introns between them shown in white boxes, with the exception of exon 23 , shown in red and purple boxes in the $\alpha$ and $\beta$ isoforms, respectively. The hatched bars at the beginning of each sequence represent the $5^{\prime}$ region of each mRNA. At the end of the STAT1A form, the hatched red bars represent the STAT1A polyadenylation site at the end of exon 25. At the end of STAT1B forms, the hatched purple bars represent the STAT1B polyadenylation site at the end of exon 23, which is longer than STAT1A exon 23. The open brackets represent the splicing events. Events shown in red predominate and are associated with the P696S STAT1 mutation.

ing intron regions in genomic DNA samples from the siblings. A homozygous nucleotide substitution at position 2,086 in exon 23 (C-> T) of STAT1 was identified in both patients. This substitution results in the proline residue in position 696 being replaced by a serine residue (P696S), between the regions of STAT1 encoding the SH2 and tail segment domains (23) (Figure 1). We found no other mutations anywhere in the coding sequence, including the 2 introns flanking exon 23. The P696S substitution was not found in 1,041 healthy controls (2,082 chromosomes) from the Centre d'Études du Polymorphisme Humain (CEPH) diversity panel (Human Genome Diversity Project-CEPH database) that we tested, suggesting that it is not an irrelevant polymorphism. Moreover, the P696 residue was found to be conserved in 27 of the 34 animal species, from fish to mammals, in which STAT1 has been sequenced (data not shown). Both parents and a clinically unaffected sibling were heterozygous for the P696S mutation. P696S is, therefore, to our knowledge a novel STAT1 allele associated with an autosomal recessive clinical phenotype, characterized by mild susceptibility to intracellular bacterial and viral diseases.

Impaired STAT1 mRNA splicing in the patients' cells. We carried out RT-PCR to investigate the corresponding mRNAs in EBVtransformed B (EBV-B) cells from a healthy control individu- al referred to as $\mathrm{C}^{+/+}$(WT/WT), P1, P2 (P696S/P696S), and a heterozygous healthy relative referred to as H (P696S/WT) (Figure $2 \mathrm{~A}$ and data not shown). Two STAT1A mRNAs were detected in the patients' cells and were sequenced: a barely detectable full-length STAT1A mRNA, with the P696S mutation in exon 23 (P696S $\alpha$ A) (Figure 2, A and B), and a much more abundant, lower MW mRNA, corresponding to STAT1A lacking exon 23 (P696S $\alpha$ B) (Figure 2, A and B). Only full-length STAT1B mRNAs carrying the P696S mutation in exon 23 (P696S $\beta$ ) were detected in the patients (Figure 2, A and B). As expected, mRNAs corresponding to the WT and P696S STAT1 forms were found in cells from H. Point mutations in exonic splice enhancers (ESEs) and in exonic splice silencers (ESSs) have been associated with the abnormal splicing out of the corresponding exons (24). ESS sequences are not well known, but ESE sequences are known to be recognized by specific serine- and arginine-rich (SR) proteins that recruit the spliceosome to the exon. In silico prediction (25) identified 3 potential ESE sites in exon 23 recognized by 2 SR proteins, SC35 and SRp40 (Figure 3A). The C2086T (P696S) nucleotide substitution would slightly decrease the binding of SC35 and increase the binding of SRp40, being markedly more positive than with the WT sequence. 
A

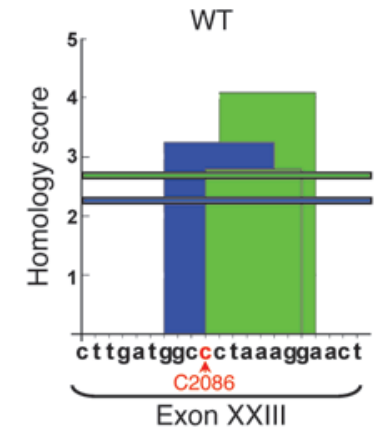

B
Xhol nt36989

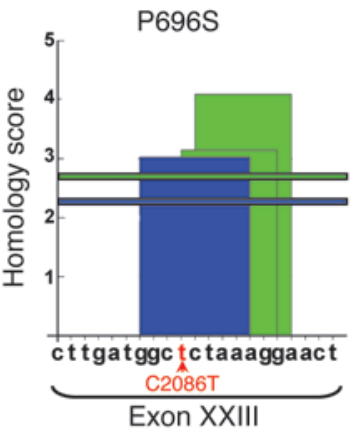

BamHI
SC35

SRp40

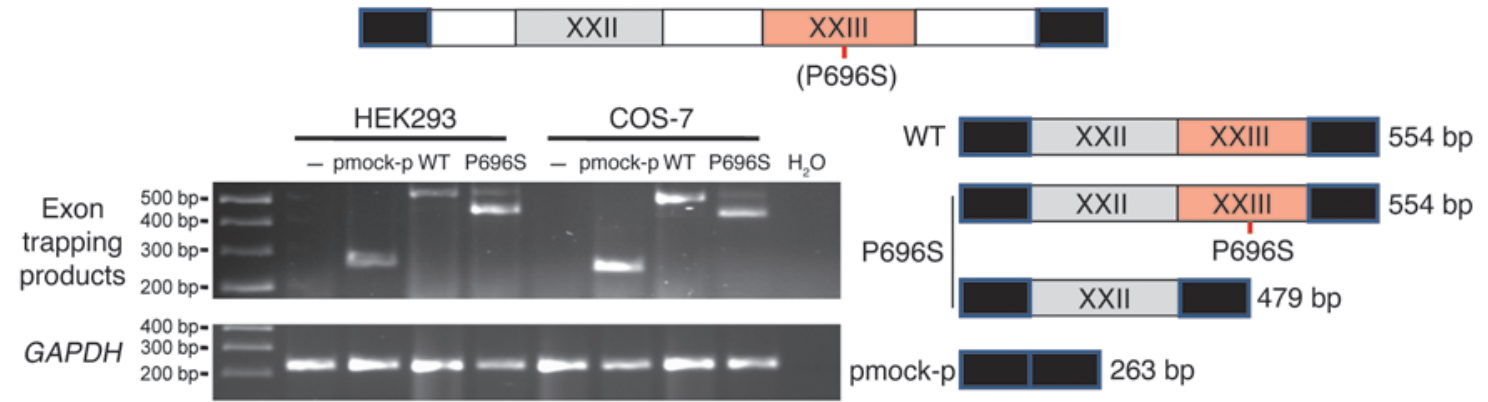

Figure 3

STAT1 P696S would be associated with an ESE defect. (A) ESE homology site in exon 23 predicted by the ESE finder program (25). Nucleotides from exon 23 are shown, with the C2086 nucleotide in the WT sequence and the C2086T substitution in the P696S sequence shown in red. The horizontal blue and green bars show the significance threshold homology score for the binding of SC35 and SRp40 proteins, respectively. The predicted binding sites of these proteins are shown as rectangles along the length of the nucleotide sequence at the height of the homology score. (B) The genomic STAT1 region from nucleotide 36989 to 38523 (NC_000002) was inserted into an exon-trapping vector using Xhol and BamH1, with or without the C2086T (P696S) nucleotide substitution. The exons are numbered in Roman numerals and shown in gray boxes, with the introns between them in white boxes, with the exception of exon 23, which is shown in a red box. The vector is shown as black boxes. HEK293 and COS-7 cells were transfected with the various constructs, the exon-trapping pSPL3 mock vector (pmock-p), or no vector (-). RNA was isolated, and the various spliced products were amplified and are shown on an agarose gel with GADPH amplification. The various products were isolated and sequenced, and the resulting sequences are also shown with corresponding exons and MW. These results are representative of 2 independent experiments.

The P696S STAT1 mutation impairs mRNA splicing. To assess the role of the P696S mutation in STAT1 mRNA splicing, we transfected HEK293 and COS-7 cells with the exon-trapping pSPL3 mock vector (pmock pSPL3) or with PSPL3 containing the STAT1 genomic region, including exons 22 and 23 and their surrounding introns, with (P696S) or without (WT) the C2086T substitution (Figure 3B). The pattern of mRNAs differed between cells transfected with the P696S and WT vectors, with abundant low-MW mRNAs lacking exon 23 found in the P696S-transfected cells only, as shown by sequencing of the corresponding cDNAs. We then transfected STAT1-deficient cells (19) with a pcDNA3-tagged V5 mock expression vector ( $\mathrm{pmock}-\mathrm{V} 5$ ) or with vectors carrying the WT $\alpha$, P696S $\alpha A$, and P696S $\alpha$ B STAT1 alleles (Figure 4). Western blotting of whole-cell extracts with an $\mathrm{N}$-terminal STAT1-specific antibody (Figure 4A) revealed the presence of proteins of similar MW in cells transfected with WT $\alpha$ and P696S $\alpha$ A STAT1, whereas no proteins were detected in cells transfected with P696S $\alpha$ B STAT1 or pmock. Full-length STAT1A and ACTIN cDNAs were detected by RT-PCR (Figure 4B) and quantified by relative quantitative PCR (Figure 4C) in transfected cells, indicating that the STAT1 alleles were correctly transfected and transcribed to levels almost similar to those in transfected cells. In conclusion, the P696S mutation was associated with the predominant splicing out of exon 23 . The
mRNA form without exon 23 was not translated, and the mRNA form containing exon 23 with the P696S mutation was translated into a protein of normal MW but present at a much lower abundance than the WT protein.

STAT1 expression levels are lower in the patients' cells. We assessed STAT1 expression in EBV-B cells from P1, P2, a heterozygous individual (referred to as $\mathrm{C}^{+/}$) (19), and a homozygous patient (referred to as $\left.\mathrm{C}^{-/}\right)$(21) for a STAT1 loss-of-expression mutation, by FACS analysis with an N-terminal STAT1-specific antibody (detecting both STAT1 isoforms) (Figure 5A). STAT1 was detected in P1 and $\mathrm{P} 2$, albeit at lower levels than in the cells of $\mathrm{H}$ and $\mathrm{C}^{+/-}$. Cells from P2 contained less STAT1 than cells from P1. Cells from 6 additional healthy controls had STAT1 levels similar to those in the cells from $\mathrm{C}^{+/+}$(data not shown). We then investigated whether the STAT1 molecules detected consisted of STAT $1 \alpha$, STAT1 $\beta$, or both, using Western blotting extracts from EBV-B cells from $\mathrm{C}^{+/+}$, $\mathrm{P} 2$, and $\mathrm{C}^{-/-}$with specific $\mathrm{N}$-terminal (detecting both isoforms) and C-terminal (detecting only STAT1 $\alpha$ ) STAT1 antibodies (Figure 5B). Both STAT $1 \alpha$ and $-\beta$ were detected, at very low levels, in cells from P2, in which STAT2 was present in normal amounts. The STAT1 $\beta$ isoform was more abundant than STAT1 $\alpha$ in the cells of P2. The P696S STAT1 mutation thus led to a reduced but not abolished expression of both STAT $1 \alpha$ and STAT $1 \beta$ in the patients' cells. 
A

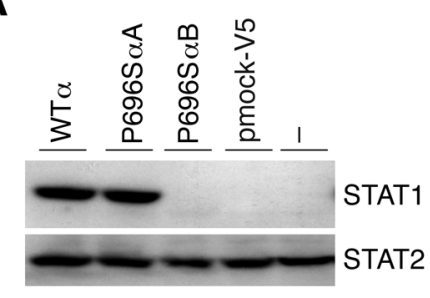

B

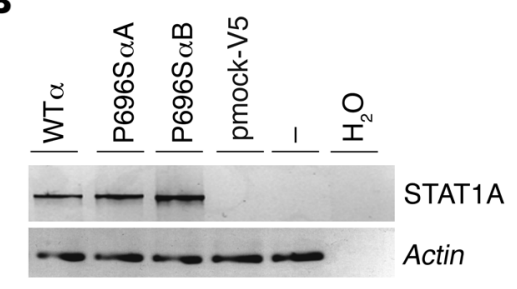

C

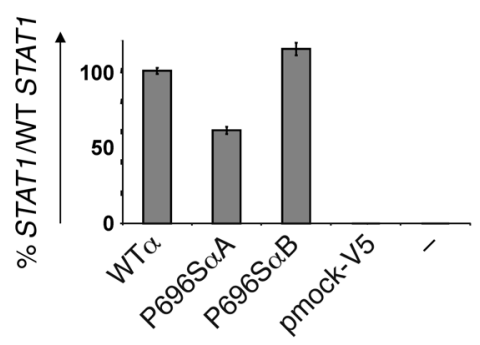

\section{Figure 4}

Only the normal splicing STAT1 P696S form is translated. Human cells completely lacking STAT1 (U3C cells) were transfected with the WT $\alpha$, $\mathrm{P} 696 \mathrm{~S} \alpha \mathrm{A}$, and P696S $\alpha \mathrm{B}$ STAT1 alleles or with the V5-tagged vector containing a mock allele (pmock-V5) or were not transfected (-). (A) These cells were subjected to Western blotting analysis with specific STAT1 and STAT2 antibodies; (B) subjected to full-length PCR amplification of the STAT1A isoform and ACTIN cDNAs; and (C) subjected to relative real-time STAT1 PCR. The results were normalized with respect to GUS mRNA and are expressed as a percentage of the amount of WT STAT1 mRNA. SD from triplication of a single experiment is indicated. These results are representative of 2 independent experiments.

Importantly, these findings demonstrated that the P696S splice mutation is leaky, as it results in the low expression of a P696S $\alpha \mathrm{A}$ isoform of normal MW in the patients' cells.

STAT1 activation is decreased in the patients' cells. We assessed STAT1 activation by investigating the phosphorylation of STAT1 Tyr701, by FACS with a specific antibody against phosphorylated Tyr701STAT1 (P-Tyr701-STAT1) in EBV-B cells from $\mathrm{C}^{+/+}, \mathrm{H}, \mathrm{P} 1, \mathrm{P} 2, \mathrm{C}^{+/-}$, and $\mathrm{C}^{-/}$, which were or were not stimulated with IFN- $\alpha$ and IFN- $\gamma$ (Figure 5C). Cells from P1 and P2 displayed lower levels of STAT1 phosphorylation in response to either IFN- $\gamma$ or IFN- $\alpha$ than cells from $\mathrm{C}^{+/+}$, whereas cells from $\mathrm{H}$ and $\mathrm{C}^{+/}$- displayed normal phosphorylation in this assay. The responses of cells from 6 other controls were similar to those of cells from $\mathrm{C}^{+/+}$(data not shown). The cells of P2 contained smaller amounts of P-STAT1 than those of P1, consistent with the modest difference in STAT1 levels revealed by flow cytometry. We then used Western blotting with specific antiP-Tyr701-STAT1, anti-P-Tyr689-STAT2, anti-STAT1, and antiSTAT2 antibodies to test for phosphorylated STAT $1 \alpha$ and STAT $1 \beta$ isoforms in $\mathrm{C}^{+/+}, \mathrm{P} 2$, and $\mathrm{C}^{-/-}$EBV-B cells stimulated with IFN- $\alpha$ or IFN- $\gamma$ (Figure 5B). Serving as a control, cells from P2 contained normal amounts of phosphorylated STAT2 (Figure 5B). However, the phosphorylation of STAT $1 \alpha$ and STAT $1 \beta$ in response to both IFN- $\alpha$ and IFN- $\gamma$ was impaired but not abolished in cells from P2. This patient, therefore, had a phenotype different from and milder than that of cells from $\mathrm{C}^{-/-}$, an individual with complete STAT1 deficiency. This defect was more pronounced for STAT $1 \alpha$ than for STAT $1 \beta$. The ratio of activated STAT $1 \alpha$ to STAT $1 \beta$ in cells from P2 was the inverse of that in $\mathrm{C}^{+/+}$. The well-known dominant-negative effect of P-STAT $1 \beta$ on P-STAT1 $\alpha$ (3) was therefore probably more pronounced in the patients' cells. In any event, the P696S mutation resulted in a partial, as opposed to complete, impairment of the phosphorylation of STAT $1 \alpha$ and STAT $1 \beta$ in response to both IFN- $\alpha$ and IFN- $\gamma$. This is consistent with the low but detectable levels of STAT $1 \alpha$ and STAT $1 \beta$ in the patients' cells.

The DNA-binding activity of STAT1 is weaker in the patients' cells. We investigated consequences of the P696S mutation for the DNAbinding activity of ISGF3 and GAF, following stimulation with IFN- $\alpha$ and IFN- $\gamma$, in EBV-B cells from $\mathrm{H}, \mathrm{C}^{+/+}, \mathrm{P} 1, \mathrm{P} 2, \mathrm{C}^{+/-}$, and $\mathrm{C}^{-/-}$, by EMSAs with radiolabeled ISRE and GAS probes, respectively (Figure 6). Following IFN- $\alpha$ stimulation, cells from $\mathrm{H}$ contained amounts of ISRE-binding proteins similar to those in the cells of $\mathrm{C}^{+/-}$and $\mathrm{C}^{+/+}$, indicating that the hypomorphic P696S STAT1 mutation, like STAT1-null mutations (19), is recessive for IFN- $\alpha-$ induced ISGF3 activation (Figure 6A). By contrast, the cells of P1 and P2 displayed a partial ISGF3 activation defect (about 66\% and $42 \%$ of normal levels for P1 and P2, respectively) (Figure 6A). Supershift experiments showed that the proteins binding to ISREs in the cells from all patients tested in this assay included STAT1, STAT2, and ISGF3- $\gamma$ (data not shown). The response to low concentrations of IFN- $\alpha$ was weaker in cells from P1 (Figure 6B). We did not investigate the cells from P2 with lower doses of IFN- $\alpha$. Following IFN- $\gamma$ stimulation, cells from $\mathrm{H}$ contained amounts of GAS-binding proteins similar to those in cells from $\mathrm{C}^{+/-}$and $\mathrm{C}^{+/+}$, demonstrating that the hypomorphic P696S STAT1 mutation, like STAT1-null mutations (19), is recessive for IFN- $\gamma$-induced GAF activation (Figure 6C). P1 and P2 cells displayed a partial GAF activation defect in response to IFN- $\gamma$ (about $41 \%$ and $12 \%$ of normal levels for P1 and P2, respectively) (Figure 6D). Supershift experiments showed that the GAS-binding proteins in all cells tested contained STAT1 (data not shown). The phenotype was also documented at low concentrations of IFN- $\gamma$ (Figure 6D). Overall, the defect in ISGF3 activation was less pronounced than that for GAF activation in the patients' cells. It is unclear why the phenotype of P2 cells was somewhat more pronounced than that of $\mathrm{P} 1$ cells, in terms of STAT1 expression, P-STAT1 activation, and DNA-binding activity. No additional genetic lesions in the STAT1 coding region in $\mathrm{P} 2$ that could account for this phenomenon were identified. Nevertheless, the 2 siblings had a similar, previously not described cellular phenotype. The P696S allele was the first STAT1 allele to our knowledge to be associated with a recessive and partial defect of both ISGF3 and GAF activation, following stimulation with IFN- $\alpha$ and IFN- $\gamma$, respectively.

The amino acid substitution $P 696$ S has no detectable functional impact. The P696 residue of STAT1 was found to be conserved in 27 species, ranging from fish to mammals, but is not conserved in other STAT molecules. P696 is located between the SH2 and P-Tyr domains, both of which are important for IFN-inducible, active STAT1 homodimerization (23). Thus, the P696S amino acid substitution may impair active STAT1/STAT1 homodimerization and possibly even active STAT1/STAT2 heterodimerization. If this were the case, the P696S mutation would be deleterious not only because of impaired mRNA splicing but also because of the amino acid substitution. We investigated this possibility by assessing STAT1/STAT2 heterodimerization, by transfect- 

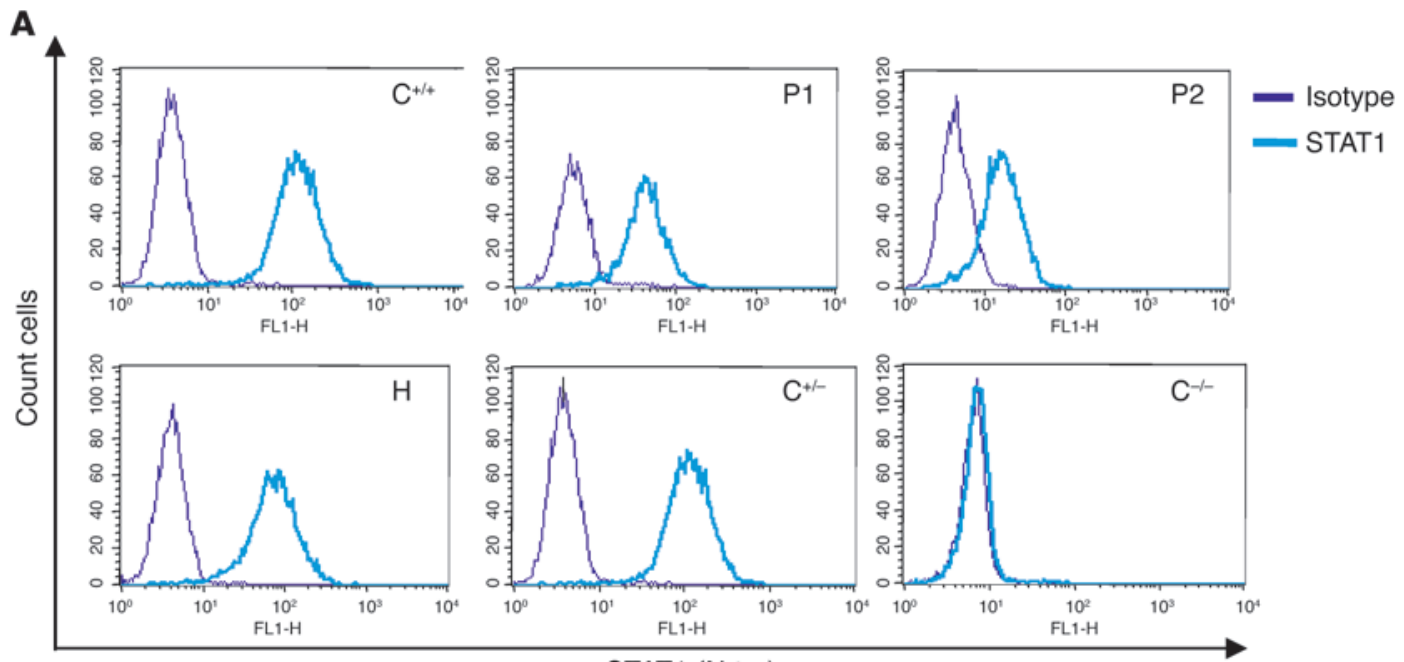

B
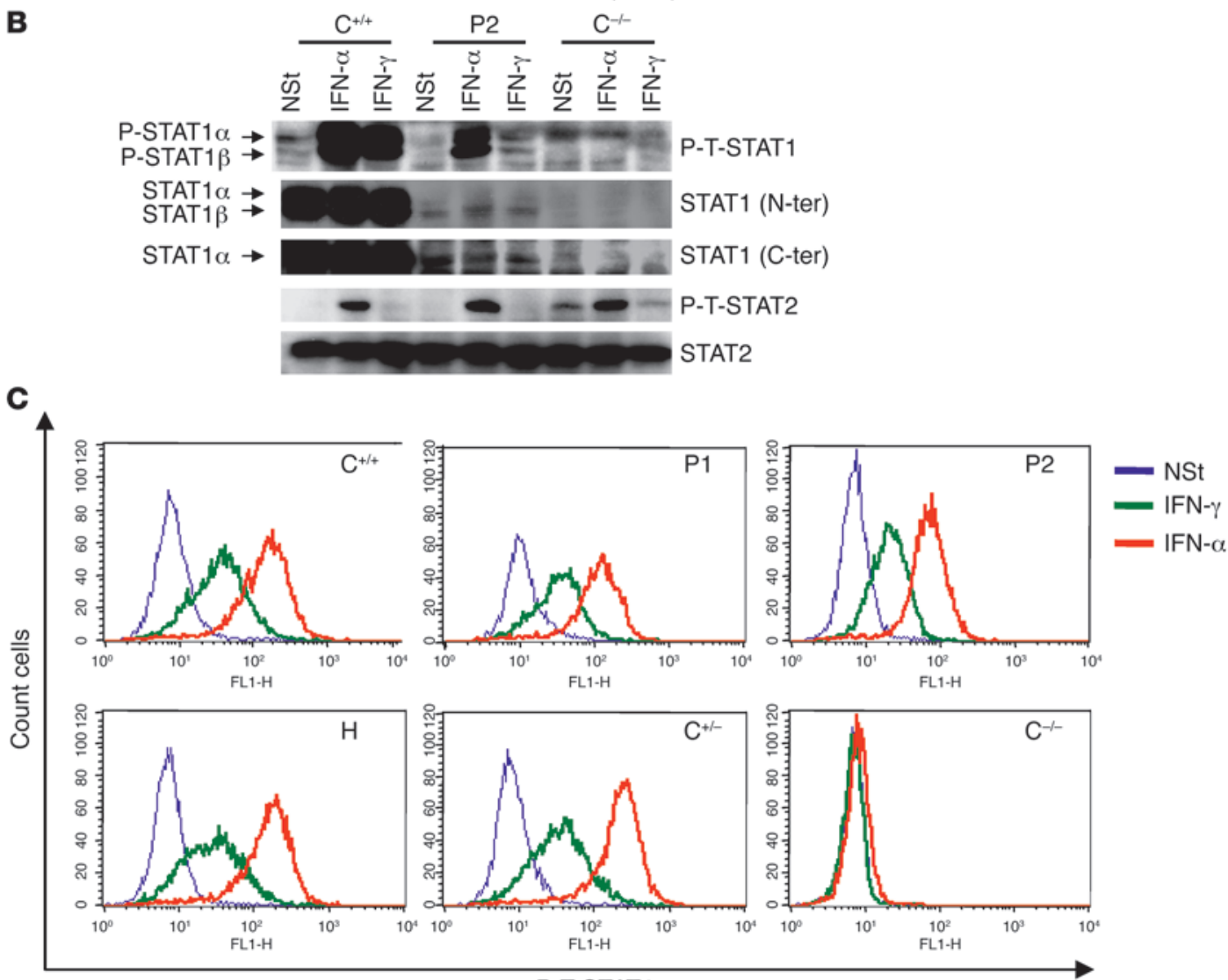

P-T-STAT1

\section{Figure 5}

STAT1 P696S is associated with low levels of STAT1 in the patients' cells. EBV-B cells from H, C $\mathrm{C}^{+/+}, \mathrm{P} 1, \mathrm{P} 2$, an individual with a single WT STAT1 allele encoding a protein $\left(\mathrm{C}^{+--}\right)$, and a patient homozygous for a mutated STAT1 allele, resulting in an absence of the protein $\left(\mathrm{C}^{-/-}\right)$, were stimulated with $10^{5} \mathrm{IU} / \mathrm{ml} \mathrm{IFN-} \alpha$ or IFN- $\gamma$ or were left unstimulated (NSt) for 30 minutes and subjected to FACS analysis (A and C) or Western blotting (B), with specific antibodies against P-Tyr701-STAT1 (P-T-STAT1) (B and C), the STAT1 N terminus (STAT1 N-ter) (A and B) and C terminus (STAT1 C-ter) (B), P-Tyr690-STAT2 (P-T-STAT2), and STAT2 (B). The results shown are representative of 2 or 3 independent experiments.

ing STAT1-deficient cells (19) with WT $\alpha$ and P696S $\alpha$ A STAT1 alleles tagged with $\mathrm{V} 5$ and then immunoprecipitating the tagged proteins from the cell lysate with specific anti-V5 and isotypic antibodies after or in the absence of stimulation with IFN- $\alpha$.
Western blotting with specific anti-STAT1 and anti-STAT2 antibodies before (Figure 7A) and after immunoprecipitation (Figure 7B) showed that similar amounts of STAT2 were coimmunoprecipitated with WT $\alpha$ and P696S $\alpha$ A V5-tagged STAT1 molecules. 
A IFN- $\alpha$
(IU/ml)

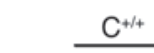
$\mathrm{C}^{+/+}$ NSt $\frac{\mathrm{P} 2}{10^{3} 10}$

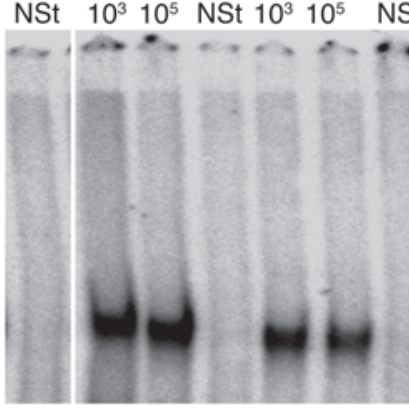

$\mathrm{H}$
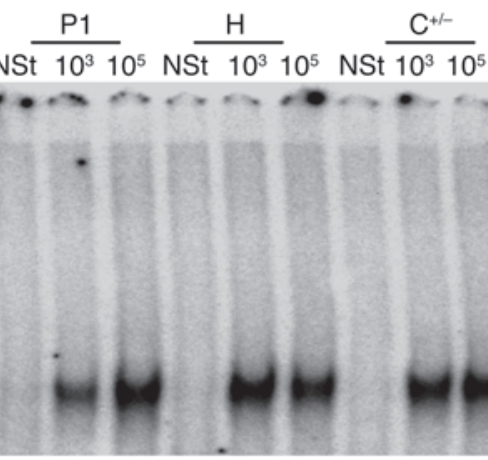

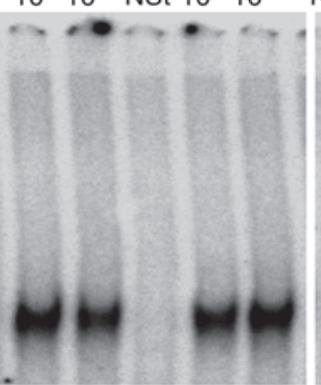

P2

\begin{abstract}
$\frac{\mathrm{P} 1}{10^{3} 10^{5}}$
\end{abstract}

\begin{abstract}
$\mathrm{H}$
\end{abstract}

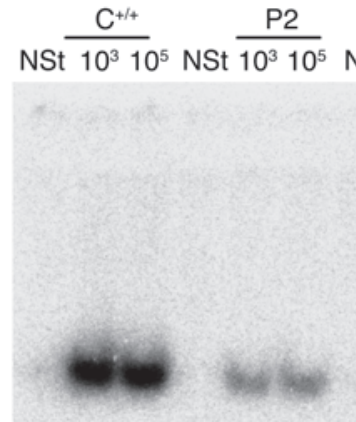

IFN- $\gamma$

(IU/ml)
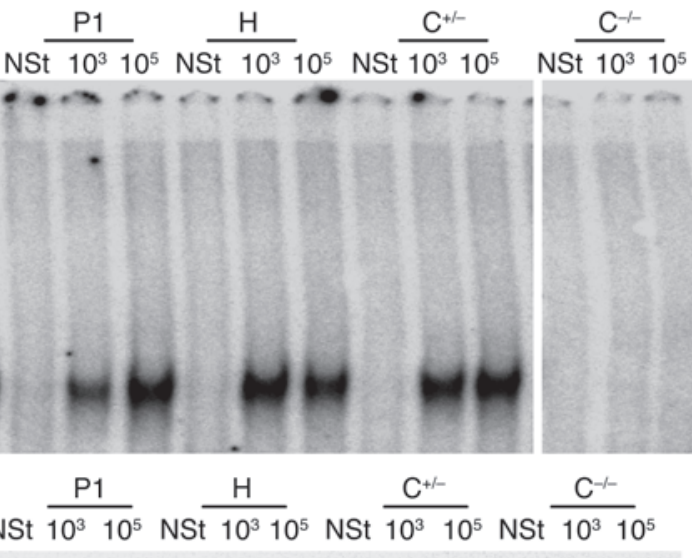

B

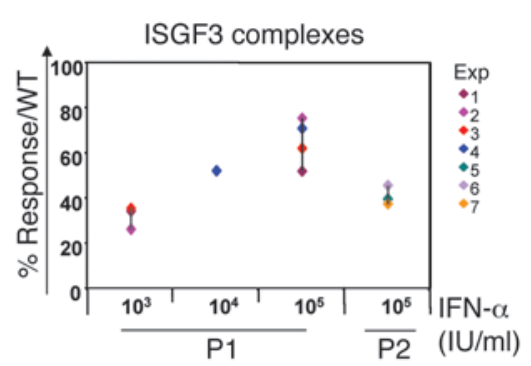

D

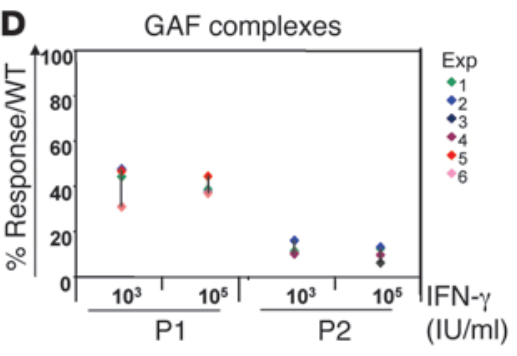

GAS

probe

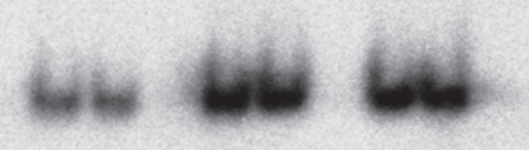

\section{Figure 6}

STAT1 P696S is associated with a partial recessive defect in the activation of both ISGF3 and GAF, following stimulation with IFN- $\alpha$ and IFN- $\gamma$. EMSA with nuclear extracts $(5 \mu \mathrm{g})$ from EBV-B cells from $\mathrm{H}, \mathrm{C}^{+/+}, \mathrm{P} 1, \mathrm{P} 2, \mathrm{C}^{+/-}$, and $\mathrm{C}^{-/-}$not stimulated or stimulated for 30 minutes with $10^{3}$ and $10^{5}$ IU/ml IFN- $\alpha(\mathbf{A})$ or IFN- $\gamma(\mathbf{C})$. Radiolabeled ISRE (A) or GAS (C) probes were used. These results are representative of 6 or 7 independent experiments (A and C). Quantification of these 6 or 7 independent experiments by Phospholmager SI (Molecular Dynamics), using the ISRE (B) or GAS (D) probe, in response to IFN- $\alpha$ (B) or IFN- $\gamma$ (D). Each independent experiment (Exp) is shown in a different color. The responses are expressed as percentages of patients' response versus a healthy control's response (defined as 100\%). Vertical lines have been drawn between maximum and minimum values. Various lanes from the same gel have been inverted in $\mathbf{A}$; white dividing lines show where the image was cut.

Thus, the P696S substitution does not substantially impair active STAT1/STAT2 heterodimerization. STAT1 homodimerization is also induced by IFN- $\alpha$, in some cases, to an even greater degree (20). We investigated STAT1 homodimerization by cotransfecting cells with WT $\alpha$ and P696S $\alpha$ A STAT1 alleles tagged either with V5 or with c-myc and immunoprecipitating the tagged proteins from the cell lysate with a specific anti-V5 antibody after (Figure $7 D$ ) or in the absence of (data not shown) stimulation with IFN- $\alpha$. Western blotting with specific anti-c-myc and anti-V5 antibodies before (Figure 7C) and after immunoprecipitation (Figure 7D) showed that similar amounts of P696S $\alpha$ A and WT $\alpha$ STAT1 were immunoprecipitated with WT $\alpha$ and P696S $\alpha$ A STAT1 molecules. Thus, the P696S substitution has no major effect on active STAT1/STAT1 homodimerization. STAT1 dimerization also depends on STAT1 Tyr701 phosphorylation after IFN- $\alpha$ stimulation, so we then assessed the consequences of the mutation for STAT1 phosphorylation in cells transfected with WT $\alpha$ and

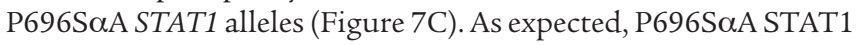
was normally phosphorylated, indicating an absence of effect of the P696S substitution on active STAT1 phosphorylation. These experiments demonstrated the absence of a detectable effect of the P696S amino acid substitution on STAT1 phosphorylation and dimerization and showed that the P696S mutation is deleterious solely because of its consequences for mRNA splicing, despite the nonconservative amino acid substitution affecting a residue of STAT1 that is conserved through evolution.
The P696S STAT1 allele impairs downstream responses to IFNs. We then used relative real-time RT-PCR (19) to assess the effects of the P696S STAT1 allele on transcription of the IFN- $\alpha-$ and IFN- $\gamma$-inducible genes myxovirus (influenza virus) resistance 1 , IFN-inducible protein p78 (mouse) (MX1), ISG15 ubiquitin-like modifier (ISG15), and IFN regulatory factor 1 (IRF1) in EBV-B cells from $\mathrm{H}, \mathrm{C}^{+/+}, \mathrm{P} 1, \mathrm{P} 2, \mathrm{C}^{+/-}$, and $\mathrm{C}^{-/-}$(Figure $8 \mathrm{~A}$; data not shown). The induction of MX1, ISG15, and IRF1 in cells from $\mathrm{H}$ and $\mathrm{C}^{+/-}$ after stimulation with IFNs was identical to that in cells from $\mathrm{C}^{+/+}$and 6 other healthy controls (data not shown). By contrast, cells from $\mathrm{P} 1$ and $\mathrm{P} 2$ displayed a partial defect in the induction of $M X 1, I S G 15$, and IRF1 in response to IFN- $\alpha$ and IFN- $\gamma$ (Figure 8A). We further assessed the protection conferred by IFN- $\alpha$ in SV-40transformed fibroblasts from $\mathrm{C}^{+/+}, \mathrm{P} 2$, and $\mathrm{C}^{-/-}$infected with herpes simplex virus (HSV) and vesicular stomatitis virus (VSV) (21) (Figure 8B). Cells from P2 were fully protected by IFN- $\alpha$, whereas cells from $\mathrm{C}^{-/-}$were not. Cells from $\mathrm{P} 2$ were also protected by lower doses of IFN- $\alpha$ (data not shown), possibly accounting for their much milder clinical phenotype for viral infections. We then assessed IFN- $\gamma$-dependent immunity in a whole-blood assay, by studying the late induction of IFN- $\gamma$ or IL-12p40 and IL-12p70 in response to live BCG vaccine, with or without IL-12 or IFN- $\gamma$ supplementation, respectively $(26,27)$ (Figure 8C and Figure 9). We were able to carry out this experiment just once for patients, and we compared the results obtained for the 2 patients and their heterozygous relative with those for a cohort of healthy controls 
A

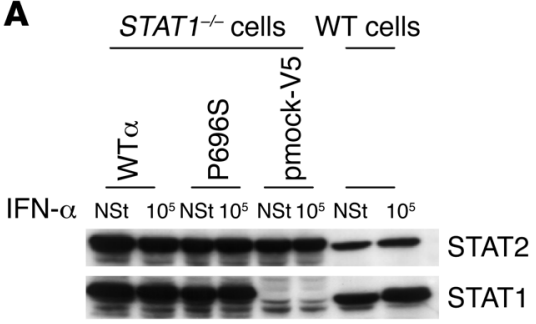

C

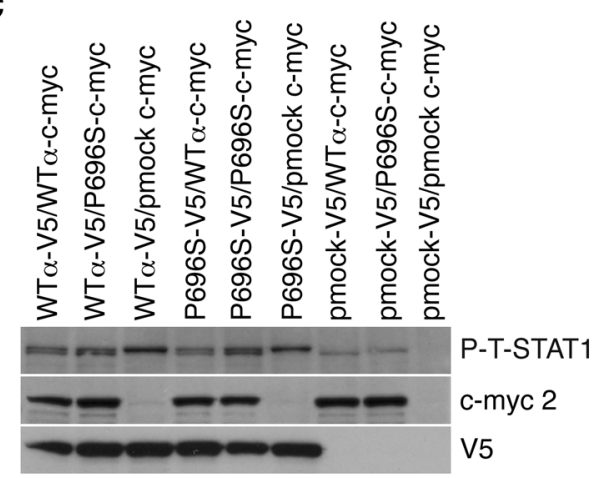

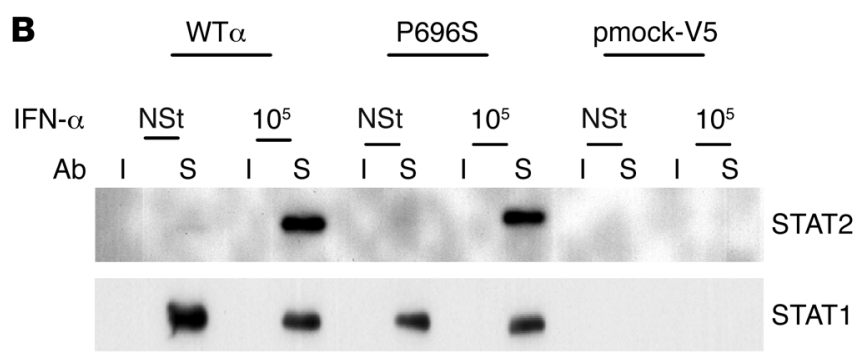

D

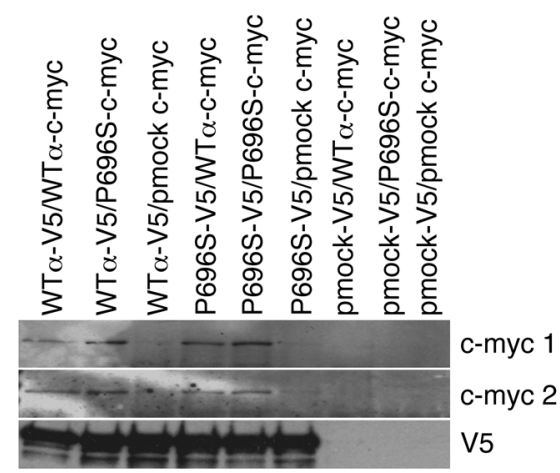

Figure 7

STAT1 P696S amino acid substitution does not affect STAT1 activation. (A and B) Immunoprecipitation with a V5-specific (S) or isotypic (I) antibody from total protein extracts $(1 \mathrm{mg})$ of parental fibrosarcoma WT cell line (2C4) or STAT1-deficient U3C fibrosarcoma cells transfected with a V5-tagged vector containing a mock allele, the WT $\alpha$, or P696S $\alpha$ A (P696S) STAT1 alleles. (A) Total protein extracts and (B) immunoprecipitates were studied by Western blotting with STAT1- and STAT2-specific antibodies. Cells were not stimulated or were stimulated for 30 minutes with

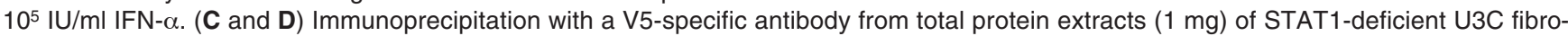
sarcoma cells cotransfected with vectors containing a mock allele, WT $\alpha$, and P696S $\alpha$ A STAT1 alleles tagged with V5 or with c-myc. (C) Total protein extracts and (D) immunoprecipitates were analyzed by Western blotting with specific antibodies against phosphorylated-Tyr701 STAT1, V5-specific, and c-myc-specific antibodies. The cells were not stimulated (data not shown) or stimulated for 30 minutes with $10^{5}$ IU//ml IFN- $\alpha$. For $\mathbf{C}$ and $\mathbf{D}$, each blot was obtained from a different gel. Each result shown is representative of 2 independent experiments (A-D).

and all previously tested patients with impaired production of IL-12 (IL-12p40 deficiency), impaired responses to IL-12 (IL-12R $\beta 1$ deficiency), or impaired responses to IFN- $\gamma$ (complete or partial defects of IFN- $\gamma$ R1, IFN- $\gamma$ R2, and STAT1) (Figure 8C and Figure 9) (26). Cells from $\mathrm{P} 1$ and $\mathrm{P} 2$, unlike those from $\mathrm{H}$, produced no IL-12 in response to BCG or BCG plus IFN- $\gamma$, like cells from patients with impaired responses to IFN- $\gamma$ and unlike those from patients with impaired IL-12 immunity. Cells from P1 and P2, unlike cells from $\mathrm{H}$, displayed a slight defect in IFN- $\gamma$ production in response to BCG or to BCG plus IL-12, like patients with impaired responses to IFN- $\gamma$ and unlike patients with defective responses to IL-12. In this whole-blood assay, the production of IL-12 upon stimulation by BCG alone depends only partly on the endogenous IFN- $\gamma$ response (Figure 8C) (26). This slight defect may be accounted for by the defect in the IFN- $\gamma$ response in patients. Homozygosity for the P696S STAT1 allele is therefore associated with early and late defects in the cellular responses to both IFN- $\alpha$ and IFN- $\gamma$.

Impaired IFN- $\lambda 1$ and IL-27 responses in STAT1-deficient patients. The activation of STAT 1 by IFN- $\lambda 1$ (IL-29) and IL-27 has recently been described. IFN- $\lambda 1$ activates ISGF3 complexes via its heterodimeric receptor, composed of the IL-10R $\beta$ and IL-28R $\alpha$ subunits (28-32). Marcello et al. (29) showed that IFN-induced protein with tetratricopeptide repeats 1 (IFIT1) is strongly induced by IFN- $\lambda 1$ in human primary hepatocytes, and Wacher et al. (33) showed that IFIT1 is induced in a STAT1-dependent manner after lymphocytic choriomeningitis virus infection in mice. We there- fore investigated whether IFN- $\lambda 1$ induced IFIT1 in EBV-B human cells and whether this induction was STAT1-dependent (Figure 10A). We observed the induction of IFIT1 in EBV-B cells from a healthy control but not in those from a STAT1-deficient patient at several time points after stimulation with IFN- $\lambda 1$. Cells from P1, with partial STAT1 deficiency, presented a partial defect in the response to IFN- $\lambda 1$ (Figure 10A). We then assessed the consequences of STAT1 deficiency for IL-27 responses. IL-27 is composed of the p28 and EBI3 subunits and drives the early differentiation of IFN- $\gamma$-producing T cells via its heterodimeric receptor, composed of the GP130 and WSX-1 subunits. Human STAT1 has been shown to be involved in IL-27 responses, but the STAT1containing complexes have not yet been characterized (34-38). We found that IL-27 did not induce ISRE-binding protein (data not shown) but did induce GAS-binding proteins containing STAT1, but not STAT2, STAT3, or STAT4 (Figure 10B), in EBV-B cells from $\mathrm{C}^{+/+}$but not in those from $\mathrm{C}^{-/-}$. The 2 patients with partial STAT1 deficiency each presented a partial defect in the response to IL-27 (Figure 10, B and C) by comparison with 5 different positive controls tested (data not shown). As IL-27 induces GAF-like complexes, we investigated STAT1-dependent IRF1 induction in response to IL-27. P1 and P2 each displayed a partial defect in IRF1 induction (Figure 10D). All these findings therefore demonstrated that recessive STAT1 deficiencies impair both the IL-27 and IFN- $\lambda 1$ signaling pathways, in addition to their effects on IFN $-\alpha / \beta$ and IFN- $\gamma$ responses. 
A

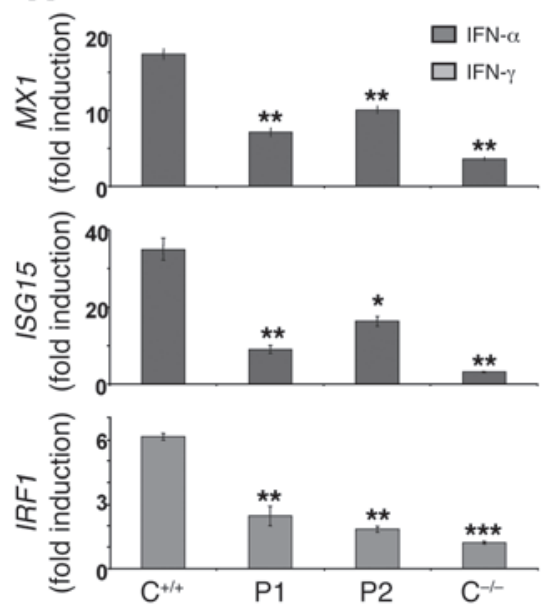

B

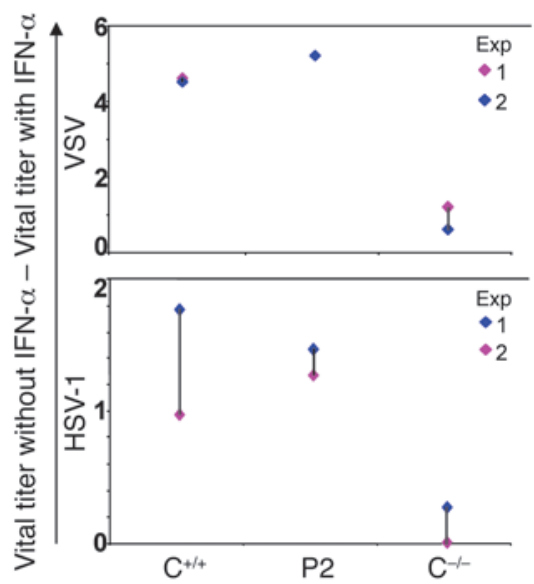

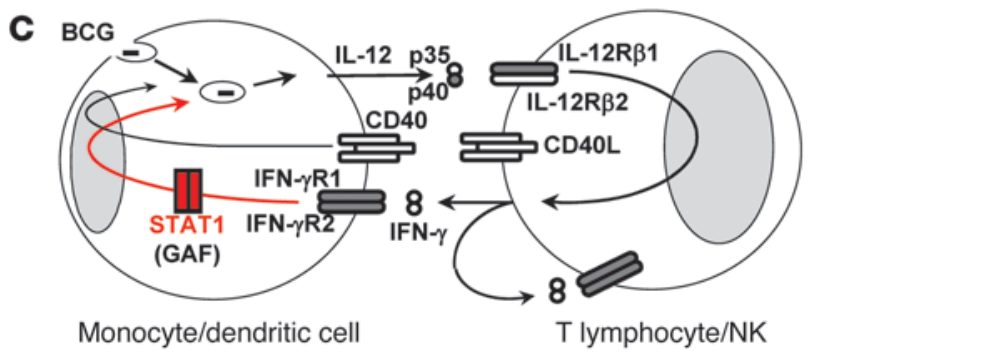

Monocyte/dendritic cell

T lymphocyte/NK

\section{Figure 8}

STAT1 P696S is associated with a partial recessive defect in late responses to IFN- $\alpha$ and IFN- $\gamma$. (A) Abundance of mRNA corresponding to genes induced by IFN- $\alpha$ and/or IFN- $\gamma$ (MX1, ISG15, and IRF1) in EBV-B cells from $\mathrm{C}^{+/+}, \mathrm{P} 1, \mathrm{P} 2$, and $\mathrm{C}^{-/-}$, either not stimulated or stimulated with $10^{5} \mathrm{IU} / \mathrm{ml}$ IFN- $\alpha$ or IFN- $\gamma$ for 2 hours. The results are normalized with respect to GUS mRNA and are expressed as multiples (fold induction) of the unstimulated value \pm SD. ${ }^{*} P<0.05$, ${ }^{* *} P<0.01$, ${ }^{* * *} P<0.001$ when compared to $\mathrm{C}^{+/+}$. The exact $P$ values are reported in Supplemental Table 2. Each result shown is representative of 2 to 3 independent experiments. (B) Skin-derived SV-40-transformed fibroblasts from $\mathrm{C}^{+/+}, \mathrm{P} 2$, and $\mathrm{C}^{-/-}$were infected with HSV-1 or VSV, with or without prior stimulation with IFN- $\alpha$ $\left(10^{5} \mathrm{IU} / \mathrm{ml}\right)$ for 24 hours. Viral titers were determined after 48 hours of infection. Each independent experiment is shown in a different color. Vertical lines have been drawn between maximum and minimum values. (C) Schematic representation of cytokine production and cooperation between monocytes/dendritic cells and T/NK cells upon live BCG stimulation. Mutant molecules from patients with MSMD defects compared with our patients are shown in gray. STAT1 and its corresponding pathway are shown in red.

\section{Discussion}

We report here a form of human STAT1 deficiency that we believe to be novel, in which the hypomorphic P696S STAT1 allele causes an autosomal recessive form of partial STAT1 deficiency. Two homozygous siblings display impaired but not abolished responses to both IFN- $\gamma$ and IFN- $\alpha / \beta$, resulting in a combination of severe but curable intracellular bacterial and viral diseases. The previously reported partial forms of STAT1 deficiency display autosomal dominant inheritance and selectively affect IFN- $\gamma$ responses in patients with MSMD $(18,19)$, whereas previously described autosomal recessive forms of STAT1 deficiency, affecting both IFN- $\gamma$ and IFN- $\alpha / \beta$ responses, are associated with a complete absence of response to IFNs and a lethal outcome $(20,21)$. One of our patients with partial STAT1 deficiency suffered from HSV infections more severe than those generally affecting healthy individuals but less severe than those in patients with complete STAT1 deficiency, one of whom is known to have died of herpes simplex encephalitis $(20,39)$. Both siblings also presented systemic salmonellosis, as do about $4 \%$ of patients with mutations in the IFN- $\gamma$ receptor genes $(27,40)$. The association of recurrent intracellular bacterial and viral diseases (caused by pathogens such as HSV and Salmonella, in particular) is typically considered to reflect innate errors of adaptive $\mathrm{T}$ cell immunity (often referred to as "combined immunodeficiencies") (41). However, it is important to consider the possibility of both complete TYK2 (22) and partial STAT1 (our observations) deficiencies as alternative, innate immunodeficiencies in such patients $(41,42)$. Unlike partial STAT1 deficiencies, complete TYK2 deficiency is associated with defects in circuits other than IFN- $\alpha / \beta$ and IL-12/IFN- $\gamma$, such as the IL- 6 and IL-10 circuits, probably accounting for the high levels of serum $\operatorname{IgE}(43)$ and the broader range of infections observed. Conversely, cellular responses to IFN- $\lambda$ and IL-27 have not been studied in TYK2 deficiency. Complete STAT1 deficiency $(20,21)$ runs a much more severe course, resembling that of patients with severe combined immunodeficiency (41).

The mechanism by which the STAT1 P696S mutation is deleterious is also interesting. We showed that the C2086T nucleotide substitution in exon 23 is associated with the predominant abnormal splicing out of exon 23 of STAT1A. C2086T either created a new ESS site or altered the existing ESE site in exon 23. Indeed, the mutation decreased the probability of SC35 and increased the probability of SRp40 binding to their predicted ESE sites containing the nucleotide 2086 in exon 23. These predicted ESE binding sites overlap, and these 2 SR proteins can have different roles $(44,45)$, so the preferential splicing out of exon 23 may be due to the preferential binding of SRp40 rather than SC35. The last exon used in STAT1B is exon 23, and this isoform is affected, but less so than the other isoform, probably because exon 23 used in STAT1B is longer and also presents the final acceptor site. The abnormally spliced STAT1 mRNAs are not translated into stable proteins. This results in much lower levels of STAT $1 \alpha$ and STAT $1 \beta$ in these patients than in patients heterozygous for loss-of-expression STAT1 mutations. We have previously shown there was no haploinsufficiency of STAT1 for both GAF and ISGF3 activations (19), and we show here that lower levels of STAT1 are detrimental. Also consistent with the clinical phenotype of both patients, the defect in ISGF3 activation is milder than that in GAF activation, probably because the limiting levels of STAT1 are less deleterious for the formation of ISGF3 complexes, which require only 1 STAT1 molecule, than for that of GAF complexes, which require 2 STAT1 molecules. Surprisingly, P696S 

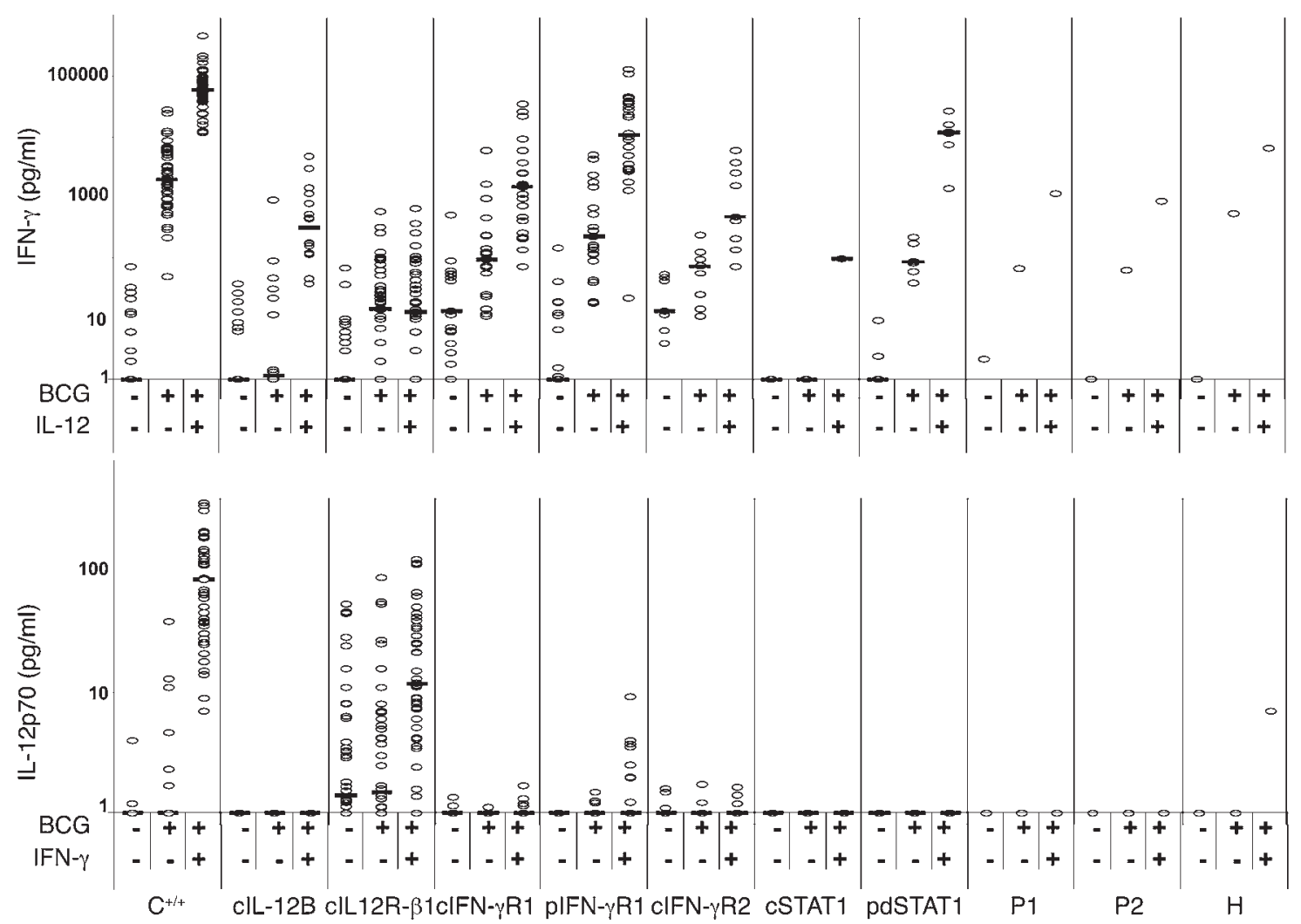

\section{Figure 9}

Whole-blood assay on patients' cells shows a defect of response to IFN- $\gamma$. Cytokine concentrations in the whole-blood supernatant from our cohort of healthy controls $\left(\mathrm{C}^{+/+}\right)$, patients with complete IL-12B deficiency (cIL-12B), patients with complete IL-12R $\beta 1$ deficiency (cIL-12R $\beta 1$ ), patients with complete IFN- $\gamma \mathrm{R} 1$ deficiency (cIFN- $\gamma \mathrm{R} 1$ ), patients with partial IFN- $\gamma \mathrm{R} 1$ deficiency (pIFN- $\gamma \mathrm{R} 1$ ), patients with complete IFN- $\gamma \mathrm{R} 2$ deficiency (cIFN- $\gamma$ R2), patients with complete STAT1 deficiency (cSTAT1), patients with partial dominant STAT1 deficiency (pdSTAT1), P1 and P2, and $\mathrm{H}$ when not stimulated or stimulated for 48 hours with live BCG alone or supplemented with IL-12 or IFN- $\gamma$. The concentrations of IFN- $\gamma$ and IL-12p70 (pg/ml) in the supernatant were determined by ELISA. Each individual is represented by an open circle, and the median is represented by a thick horizontal bar.

STAT1, when appropriately expressed, functioned normally, and the missense mutation did not impair STAT1 activation. The mutation is deleterious solely because it impairs mRNA splicing. It is hypomorphic because of the residual expression of a full-length mRNA encoding a functional STAT1 protein. These observations demonstrate that the STAT1 P696S mutation is a hypomorphic splicing mutation, resulting in a recessive form of partial STAT1 deficiency.

Finally, we studied the involvement of STAT1 in cellular responses to the newly described cytokines IFN- $\lambda 1$ (IL-29) and IL-27. Human IFN- $\lambda 1$ has been implicated in immunity to several viruses in vitro, including the hepatitis B and C viruses $(28,29)$, hantavirus $(31)$, VSV, and encephalomyocarditis virus $(30,46)$. In most human cell types studied, IFN- $\lambda 1$ activates ISGF3 less efficiently than IFN- $\alpha$, but IFN- $\lambda 1$ is as protective as IFN- $\alpha$ against hepatitis B and C in hepatocytes $(28,29)$. We showed that IFIT1 was induced in a STAT1dependent manner by IFN- $\lambda 1$ in human EBV-B cells. Patients with partial and complete STAT1 deficiency displayed impaired and abolished responses to IFN- $\lambda 1$, respectively. Impaired IFN- $\lambda$ (IL-28 and IL-29) immunity may therefore favor the viral diseases observed in patients with complete or partial STAT1 deficiency. Moreover, IL27ra knockout mice are susceptible to intracellular parasites, such as Leishmania major (47), and intracellular bacteria, such as Listeria monocytogenes (48). Salmonella also induces IL-27 in human bone marrow-derived macrophages (49). We showed that IL-27 induced STAT1-containing GAS-binding proteins and STAT1-dependent IRF1 transcription and that both these phenomena were absent from the cells of patients with complete STAT1 deficiency. The 2 siblings with partial STAT1 deficiency and salmonellosis showed impaired but not abolished responses to IL-27. It is therefore possible that impaired IL-27-mediated immunity may have contributed to the development of salmonellosis (50). The respective contributions of human IFN- $\alpha / \beta$, IFN- $\gamma$, IFN- $\lambda 1$, and IL-27 to the infectious phenotypes of STAT1-deficient patients remain unclear, and further studies are required to clarify the role of these cytokines.

\section{Methods}

Patients. We investigated 2 siblings with bacterial and viral diseases born to consanguineous parents originating from and living in Israel (Figure 1B). P1 developed severe, recurrent Salmonella group D infection at the age of 2 years, with bacteremia and cholecystitis. He never received prophylaxis. At the age of 9 years, he suffered a new episode of salmonellosis. P2, his younger sister, developed meningitis caused by Salmonella group D, recurrent pneumonia, and asthma; she also had a single episode of sinusitis and osteomyelitis in the iliac bone and a urinary tract infection. P2 also developed viral diseases, requiring hospitalization, caused by cytomegalovirus and varicella-zoster virus, with secondary cutaneous infection. Neither 
$\mathbf{A}$

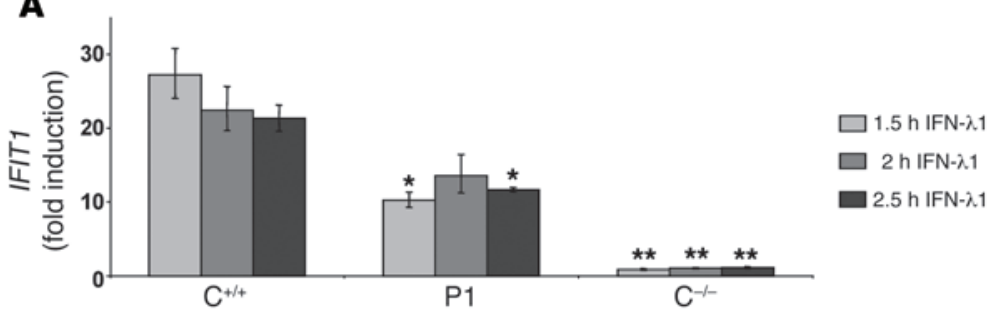

B

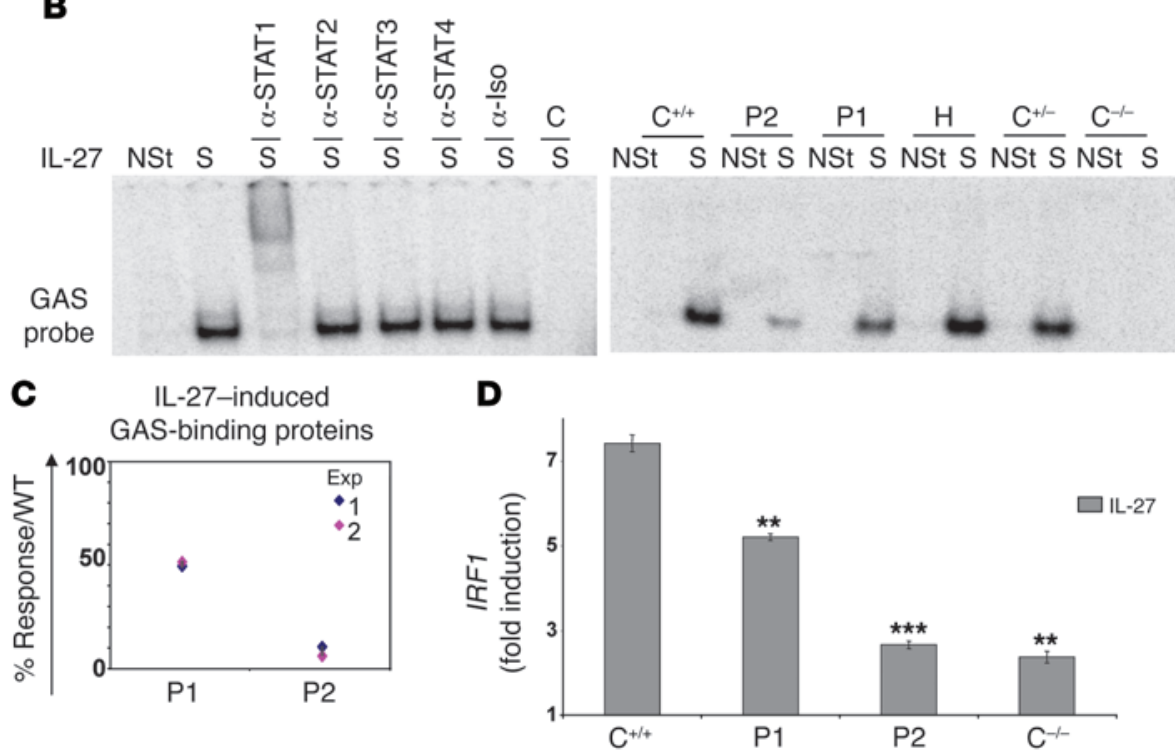

Figure 10

The IL-27 and IFN- $\lambda 1$ pathways are STAT1 dependent. (A) IFIT1 mRNA in EBV-B cells from $\mathrm{C}^{+/+}$, P1, and C $\mathrm{C}^{-/-}$after stimulation for 1.5, 2 , and 2.5 hours with $20 \mathrm{ng} / \mathrm{ml}$ IFN- $\lambda 1$ or no stimulation. Results are normalized with respect to GUS mRNA and are expressed as multiples (fold induction) of the unstimulated value $\pm \mathrm{SD}$. ${ }^{*} P<0.05,{ }^{* *} P<0.01$ when compared to $\mathrm{C}^{+/+}$(Supplemental Table 2$)$. The experiment shown is representative of 3 independent experiments. (B) EMSA with nuclear extracts $(5 \mu \mathrm{g})$ from EBV-B cells from $\mathrm{H}, \mathrm{C}^{+/+}, \mathrm{P} 1, \mathrm{P} 2, \mathrm{C}^{+/-}$, and $\mathrm{C}^{-/-}$not stimulated or stimulated (S) for 30 minutes with $100 \mathrm{ng} / \mathrm{ml} \mathrm{IL-27.} \mathrm{(B} \mathrm{and} \mathrm{C)} \mathrm{Radiolabeled} \mathrm{GAS} \mathrm{probes} \mathrm{were} \mathrm{used.} \mathrm{For} \mathrm{supershift} \mathrm{experiments,} \mathrm{IL-27-stimulated} \mathrm{nuclear}$ extracts from $\mathrm{C}^{+/+}$were first incubated with antibodies specific for STAT1, STAT2, STAT3, STAT4, the corresponding isotypic antibodies (Iso), or with a non-radiolabeled probe (C). (C) The experiments shown are representative of 2 to 3 independent experiments. Quantification by PhosphoImager SI (Molecular Dynamics), using the GAS probe, of the response to $100 \mathrm{ng} / \mathrm{ml} \mathrm{IL-27.} \mathrm{Each} \mathrm{independent} \mathrm{experiment} \mathrm{is} \mathrm{shown} \mathrm{in} \mathrm{a} \mathrm{different}$ color. The responses are expressed as percentages of the positive control response (taken as 100\%). (D) Abundance of IRF1 mRNA in EBV-B

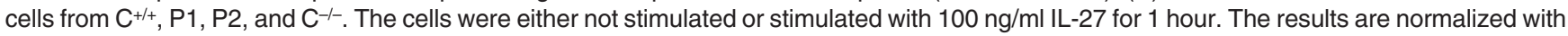
respect to GUS mRNA and are expressed as multiples (fold induction) of the unstimulated value \pm SD. ${ }^{* *} P<0.01$, ${ }^{* * *} P<0.001$ when compared to $\mathrm{C}^{+/+}$. The exact $P$ values are reported in Supplemental Table 2. The experiment shown is representative of 2 independent experiments.

patient had been vaccinated with BCG. P2 was checked for serum IgE levels at the age of 5 years ( 41 units $/ \mathrm{ml}$ ) and at the age of 11 years $(155.9$ units $/ \mathrm{ml})$. These levels were considered normal. P2 also suffered from HSV gingivostomatitis. Both patients were alive at the time of publication and currently live and are followed in Israel, where their informed consent for participation in this study was obtained in accordance with local regulations and with the approval of the IRB of Hadassah University. The experiments described here were conducted in France, in accordance with local regulations and with the approval of the IRB of Necker Enfants Malades Hospital.

Cell culture and stimulation, DNA and RNA extraction, and PCR sequencing and sequence alignment. EBV-B, SV-40-transformed fibroblast cell lines, and the STAT1-deficient U3C-derived cell line were cultured as previously described (19). Cells were stimulated with the indicated doses of IFN- $\gamma$ (Imukin; Boehringer Ingelheim), IFN- $\alpha 2 \mathrm{~b}$ (Intron A; Schering-Plough), IL-27 (R\&D Systems), and IFN- $\lambda 1$ (R\&D Systems). Genomic DNA and total RNA were extracted from cell lines and from fresh blood cells, as previously described (19). Genomic DNA and cDNA were amplified and sequenced as previously described (19). Primers and PCR conditions are reported in Supplemental Table 1. The known STAT1 protein sequences from Ensembl (http://www.ensembl.org) were aligned using the Multiple Sequence Alignment CLUSTALW (http://align.genome.jp).

Determination of $m R N A$ levels by relative real-time PCR. Total RNA was extracted with TrizoL reagent (Invitrogen) from EBV-B cells left unstimulated or stimulated with $10^{5} \mathrm{IU} / \mathrm{ml}$ IFN- $\alpha$ or IFN- $\gamma$ or with $100 \mathrm{ng} / \mathrm{ml}$

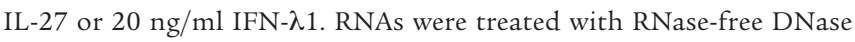
(Roche Diagnostics) and cleaned by passage through an RNAeasy column (Qiagen). RNAs were then reverse-transcribed directly with Oligo-dT, using the TaqMan Reverse Transcription kit (Applied Biosystems, Roche), for the determination of MX1, ISG15, IRF1, and IFIT1 mRNA levels, using TaqMan probes (Applied Biosystems) for these genes. The results were normalized with respect to the values obtained for the endogenous GUS CDNA.

Statistics. Data are expressed as mean \pm SD. Differences with the WT control in relative quantitative PCR were analyzed with the unpaired, 2-tailed Student's $t$ test, using GraphPad Software (http://www.graphpad. 
com/quickcalcs). The $P$ values are indicated in the figure legends, and their exact values are reported in Supplemental Table 2. A $P$ value of less than 0.05 was considered statistically significant.

Expression vectors and transfection. The WT $\alpha$ and mutated P696S $\alpha$ B STAT1 alleles were inserted into a V5-topo-pcDNA3 vector, according to the manufacturer's instructions. The mutated P696S $\alpha$ A STAT1 was obtained using the QuikChange Site-Directed Mutagenesis kit (Stratagene), following the manufacturer's instructions. The c-myc-tagged WT $\alpha$ STAT1A pcDNA6 vector was provided by the Schreiber laboratory and the mutated P696SaA STAT1 allele was obtained with the QuikChange Site-Directed Mutagenesis kit (Stratagene), following the manufacturer's instructions. U3C cells were transfected with 1 of the various STAT1 V5- or c-myc-tagged vectors or an insert-less V5or c-myc-tagged vector (pmock), using a calcium phosphate-based transfection kit (Invitrogen), according to the manufacturer's instructions.

Exon trapping. We amplified STAT1 genomic DNA from nucleotide 36989 to 38523 (NC_000002) with the following primers: forward, 5'cgcCTCGA G(XhoI)cctccctttattttccetga 3'; reverse, 5'cgcGGATCC(BamHI)ttcagctgtgat ggcgatag $3^{\prime}$. PCR products and empty pSPL3 plasmids (provided by Ralph Burkhardt, The Rockefeller University) were digested with XhoI and BamH1 (New England BioLabs). Plasmids were then dephosphorylated, and $50 \mathrm{ng}$ of purified plasmids and $250 \mathrm{ng}$ of purified PCR products were ligated with T4 ligase (New England BioLabs). The ligation products were then used to transform competent HB101 cells (Promega). Positive clones were identified by double enzyme digestion and sequencing. The Stratagene QuikChange Site-Directed Mutagenesis kit was used to introduce the P696S STAT1 point mutation into the WT clones. Plasmids were used to transfect HEK293 and COS-7 cells, with the Lipofectamine kit (Invitrogen), following manufacturer's instructions. Cells were harvested 24 hours after transfection, and total RNA was extracted as previously described. cDNA synthesis and PCR were carried out according to the kit manufacturer's instructions, as previously described (19). The SD6 (TCTGAGTCACCTGGACAACC) and SA2 (ATCTCAGTGGTATTTGTGAGC) primers for PSPL3 plasmids were used for amplifications for the analysis of potential splicing. $G A D P H$ was amplified as previously described (51). All of these steps were performed according to the kit manufacturer's instructions.

EMSA. EMSA was carried out as previously described (19). Briefly, cells were stimulated as indicated above. We incubated $5 \mu \mathrm{g}$ of nuclear extract with ${ }^{32}$-labeled ( $\alpha$ dATP) GAS (from the FCGR1 promoter) or ISRE (from the ISG15 promoter) probes and subjected the mixture to electrophoresis in a polyacrylamide gel. The unlabeled probe was incubated with the cells for 30 minutes at $4^{\circ} \mathrm{C}$ before addition of the radiolabeled probe. Supershift experiments were carried out as previously described (19). The antibodies used were directed specifically against STAT1 (catalog no. sc-345; Santa Cruz Biotechnology Inc.), STAT2 (catalog no. sc-476; Santa Cruz Biotechnology Inc.), STAT3 (catalog no. sc-7179; Santa Cruz Biotechnology Inc.), STAT4 (catalog no. sc-486; Santa Cruz Biotechnology Inc.), or p48 (catalog no. sc-496; Santa Cruz Biotechnology Inc.), and the corresponding isotypic antibodies were obtained from Santa Cruz Biotechnology Inc. All EMSA quantifications have been made using a PhosphoImager SI (Molecular Dynamics).

Immunoprecipitation and Western blotting. Cells were lysed by incubation in the following buffer (50 mM Tris- $\mathrm{HCl}$, pH 8.00, $150 \mathrm{mM} \mathrm{NaCl}, 0.5 \%$ NP-40, $10 \%$ glycerol, $1 \mathrm{mM}$ EDTA, $10 \mathrm{mM} \mathrm{NaF}, 1 \mathrm{mM}$ sodium orthovanadate, $750 \mu \mathrm{M}$ DTT, $1 \mathrm{mM}$ PMSF, and $10 \mu \mathrm{g} / \mathrm{ml}$ leupeptin and aprotinin) for 30 minutes at $4^{\circ} \mathrm{C}$. The lyses were then centrifuged at $10,000 \mathrm{~g}$ for 10 minutes at $4^{\circ} \mathrm{C}$. The supernatants were either processed directly for Western blotting or subjected to immunoprecipitation with SigmaPrep spin columns (MC1000; Sigma-Aldrich), $2 \mu \mathrm{g}$ of anti-V5 antibody (Invitrogen), and $30 \mu \mathrm{l}$ of protein $\mathrm{G}$ (P-3296; Sigma-Aldrich). We washed the immunoprecipitates according to the manufacturer's instructions and processed them for Western blotting. Western blotting was performed as previously described (19), with antibodies against P-STAT1 (catalog no. 9171; Cell Signaling Technology), N-terminal STAT1 (catalog no. 610116; BD Biosciences - Transduction Laboratories), C-terminal STAT1 (catalog no. sc-345; Santa Cruz Biotechnology Inc.), Tyr690STAT2 (catalog no. sc-21689; Santa Cruz Biotechnology Inc.), STAT2 (catalog no. sc-476; Santa Cruz Biotechnology Inc.), V5 (Invitrogen), c-myc 1 (clone 4A6; Upstate), and c-myc 2 (catalog no. 2272; Cell Signaling Technology).

Flow cytometry. One million EBV-B cells (per point) were incubated for 1 hour in $15 \mathrm{ml}$ of RPMI supplemented with $1 \%$ fetal calf serum. They were then activated by incubation in $0.5 \mathrm{ml} 10^{5} \mathrm{IU} / \mathrm{ml}$ IFN- $\alpha$ or IFN- $\gamma$ for 30 minutes at $37^{\circ} \mathrm{C}$. Activation was stopped by adding $1 \mathrm{ml}$ of cold $1 \mathrm{x}$ PBS. The cells were then incubated with 4\% PFA for 10 minutes at room temperature, washed in $1 \times \mathrm{PBS}$, and incubated with $100 \%$ methanol at $4^{\circ} \mathrm{C}$ for 10 minutes. The cells were washed twice and incubated with $1 \mathrm{x}$ PBS plus $1 \% \mathrm{SAB}$ plus $0.1 \%$ saponin at $4^{\circ} \mathrm{C}$ for 10 minutes. They were then washed and incubated for 1 hour at $4^{\circ} \mathrm{C}$ with antibodies against P-Tyr701-STAT1 (catalog no. 612132; BD Biosciences - Transduction Laboratories) or STAT1 (catalog no. 610116; BD Biosciences - Transduction Laboratories) or with the corresponding isotypic antibodies. The cells were then washed and incubated with a secondary antibody coupled with Alexa Fluor G488 (Molecular Probes, Invitrogen) for 20 minutes at $4^{\circ} \mathrm{C}$ and washed 3 times, and the signals were analyzed with a FACScan machine (Becton Dickinson), using CELLQuest software (Becton Dickinson).

Whole-blood assay of the IL-12-IFN- $\gamma$ circuit. Whole-blood assays were performed as previously described (26). Heparin-treated blood samples from healthy controls, $\mathrm{P} 1, \mathrm{P} 2$, and $\mathrm{H}$ were stimulated in vitro with BCG alone or with BCG plus IFN- $\gamma$ or IL-12 (R\&D Systems). Supernatants were collected after 48 hours of stimulation, and ELISA was performed with specific antibodies directed against IFN- $\gamma$, IL-12p70, or IL-12p40, using the human Quantikine IL-12p70 HS and IL-12p40 from R\&D Systems and the human Pelipair IFN- $\gamma$ kit from Sanquin, according to the manufacturer's instructions.

Viral assays. Viral assays were performed as previously described (21). Briefly, skin-derived SV-40-transformed fibroblasts were left untreated or were treated with $10^{5} \mathrm{IU} / \mathrm{ml} \mathrm{IFN}-\alpha$ for 24 hours. They were then infected by incubation with various titers of HSV or VSV, and after 48 hours, viral titers were determined by visualizing cell lysis.

\section{Acknowledgments}

We thank Anne Puel and Horst von Bernuth for helping to optimize experimental conditions. We thank Claire Fieschi, Orchidée Filipe-Santos, and Claude-Agnès Reynaud for helpful discussions. We thank Lucile Jannière, Yoann Rose, Martine Courat, Michèle N'Guyen, Catherine Bidalled, Tony Leclerc, and Guy Brami for technical and secretarial assistance and all members of the Laboratory of Human Genetics of Infectious Diseases for helpful discussions. Ariane Chapgier was supported by the Fondation pour la Recherche Médicale (FRM). Laboratory of Human Genetics of Infectious Diseases is supported in part by grants from the Schlumberger Foundation, the BNP Paribas Foundation, the March of Dimes, the Dana Foundation, the Agence Nationale pour la Recherche (ANR), and the EU grant QLK2-CT-2002-00846. Jean-Laurent Casanova is an International Scholar of the Howard Hughes Medical Institute.

Received for publication August 8, 2008, and accepted in revised form March 18, 2009.

Address correspondence to: Jean-Laurent Casanova, Laboratory of Human Genetics of Infectious Diseases, The Rockefeller University, 1230 York Avenue, Box 163, New York, New York 10065, USA. Phone: (212) 327-7331; Fax: (212) 327-7330; E-mail: jean-laurent. casanova@rockefeller.edu. 
1. Platanias, L.C. 2005. Mechanisms of type-I- and type-II-interferon-mediated signalling. Nat. Rev. Immunol. 5:375-386.

2. Schindler, C., Levy, D.E., and Decker, T. 2007. JAKSTAT signaling: from interferons to cytokines. J. Biol. Chem. 282:20059-20063.

3. Zakharova, N., et al. 2003. Distinct transcriptional activation functions of STAT1alpha and STAT1 beta on DNA and chromatin templates. J. Biol. Chem. 278:43067-43073.

4. Alvarez, G.R., Zwilling, B.S., and Lafuse, W.P. 2003. Mycobacterium avium inhibition of IFN-gamma signaling in mouse macrophages: Toll-like receptor 2 stimulation increases expression of dominant-negative STAT1 beta by mRNA stabilization. J. Immunol. 171:6766-6773.

5. Banninger, G., and Reich, N.C. 2004. STAT2 nuclear trafficking. J. Biol. Chem. 279:39199-39206.

6. Mao, X., et al. 2005. Structural bases of unphosphorylated STAT1 association and receptor binding. Mol. Cell. 17:761-771.

7. Zhong, M., et al. 2005. Implications of an antiparallel dimeric structure of nonphosphorylated STAT1 for the activation-inactivation cycle. Proc. Natl. Acad. Sci. U. S. A. 102:3966-3971.

8. Vinkemeier, U. 2004. Getting the message across, STAT! Design principles of a molecular signaling circuit. J. Cell Biol. 167:197-201.

9. Meraz, M.A., et al. 1996. Targeted disruption of the Stat 1 gene in mice reveals unexpected physiologic specificity in the JAK-STAT signaling pathway. Cell. 84:431-442.

10. Durbin, J.E., Hackenmiller, R., Simon, M.C., and Levy, D.E. 1996. Targeted disruption of the mouse Stat 1 gene results in compromised innate immunity to viral disease. Cell. 84:443-450.

11. Gil, M.P., et al. 2001. Biologic consequences of Stat1-independent IFN signaling. Proc. Natl. Acad. Sci. U. S. A. 98:6680-6685.

12. Shresta, S., et al. 2005. Critical roles for both STAT1-dependent and STAT1-independent pathways in the control of primary dengue virus infection in mice. J. Immunol. 175:3946-3954.

13. Sugawara, I., Yamada, H., and Mizuno, S. 2004. STAT1 knockout mice are highly susceptible to pulmonary mycobacterial infection. Tohoku J. Exp. Med. 202:41-50.

14. Rothfuchs, A.G., et al. 2006. STAT1 regulates IFNalpha beta- and IFN-gamma-dependent control of infection with Chlamydia pneumoniae by nonhemopoietic cells. J. Immunol. 176:6982-6990.

15. Rosas, L.E., et al. 2006. Cutting edge: STAT1 and T-bet play distinct roles in determining outcome of visceral leishmaniasis caused by Leishmania donovani. I. Immunol. 177:22-25.

16. Gavrilescu, L.C., Butcher, B.A., Del Rio, L., Taylor, G.A., and Denkers, E.Y. 2004. STAT1 is essential for antimicrobial effector function but dispensable for gamma interferon production during Toxoplasma gondii infection. Infect. Immun. 72:1257-1264.

17. Lieberman, L.A., Banica, M., Reiner, S.L., and Hunter, C.A. 2004. STAT1 plays a critical role in the regulation of antimicrobial effector mechanisms, but not in the development of Th1-type responses during toxoplasmosis. J. Immunol. 172:457-463.

18. Dupuis, S., et al. 2001. Impairment of mycobacterial but not viral immunity by a germline human STAT1 mutation. Science. 293:300-303.
19. Chapgier, A., et al. 2006. Novel STAT1 alleles in otherwise healthy patients with mycobacterial disease. PLoS Genet. 2:e131.

20. Dupuis, S., et al. 2003. Impaired response to interferon-alpha/beta and lethal viral disease in human STAT1 deficiency. Nat. Genet. 33:388-391.

21. Chapgier, A., et al. 2006. Human complete Stat-1 deficiency is associated with defective type I and II IFN responses in vitro but immunity to some low virulence viruses in vivo. J. Immunol. 176:5078-5083.

22. Minegishi, Y., et al. 2006. Human tyrosine kinase 2 deficiency reveals its requisite roles in multiple cytokine signals involved in innate and acquired immunity. Immunity. 25:745-755.

23. Chen, X., et al. 1998. Crystal structure of a tyrosine phosphorylated STAT-1 dimer bound to DNA. Cell. 93:827-839.

24. Cartegni, L., Chew, S.L., and Krainer, A.R. 2002. Listening to silence and understanding nonsense: exonic mutations that affect splicing. Nat. Rev. Genet. 3:285-298.

25. Cartegni, L., Wang, J., Zhu, Z., Zhang, M.Q., and Krainer A.R. 2003. ESEfinder 3.0: a web resource to identify exonic splicing enhancers. http:// rulai.cshl.edu/cgi-bin/tools/ESE3/esefinder. cgi? process $=$ home.

26. Feinberg, J., et al. 2004. Bacillus Calmette Guerin triggers the IL-12/IFN-gamma axis by an IRAK-4and NEMO-dependent, non-cognate interaction between monocytes, NK, and T lymphocytes. Eur. J. Immunol. 34:3276-3284.

27. Filipe-Santos, O., et al. 2006. Inborn errors of IL-12/23- and IFN-gamma-mediated immunity: molecular, cellular, and clinical features. Semin. Immunol. 18:347-361.

28. Doyle, S.E., et al. 2006. Interleukin-29 uses a type 1 interferon-like program to promote antiviral responses in human hepatocytes. Hepatology. 44:896-906.

29. Marcello, T., et al. 2006. Interferons alpha and lambda inhibit hepatitis $\mathrm{C}$ virus replication with distinct signal transduction and gene regulation kinetics. Gastroenterology. 131:1887-1898.

30. Kotenko, S.V., et al. 2003. IFN-lambdas mediate antiviral protection through a distinct class II cytokine receptor complex. Nat. Immunol. 4:69-77.

31. Stoltz, M., Ahlm, C., Lundkvist, A., and Klingstrom, J. 2007. Lambda interferon (IFN-lambda) in serum is decreased in hantavirus-infected patients, and in vitro-established infection is insensitive to treatment with all IFNs and inhibits IFN-gamma-induced nitric oxide production. J. Virol. 81:8685-8691.

32. Dumoutier, L., et al. 2004. Role of the interleukin (IL)-28 receptor tyrosine residues for antiviral and antiproliferative activity of IL-29/interferon-lambda 1: similarities with type I interferon signaling. J. Biol. Chem. 279:32269-32274.

33. Wacher, C., et al. 2007. Coordinated regulation and widespread cellular expression of interferon-stimulated genes (ISG) ISG-49, ISG-54, and ISG-56 in the central nervous system after infection with distinct viruses. J. Virol. 81:860-871.

34. Takeda, A., et al. 2003. Cutting edge: role of IL-27/ WSX-1 signaling for induction of T-bet through activation of STAT1 during initial Th1 commitment. J. Immunol. 170:4886-4890.

35. Kastelein, R.A., Hunter, C.A., and Cua, D.J. 2007.
Discovery and biology of IL-23 and IL-27: related but functionally distinct regulators of inflammation. Annu. Rev. Immunol. 25:221-242.

36. Yoshimura, T., et al. 2006. Two-sided roles of IL27: induction of Th1 differentiation on naive CD4+ T cells versus suppression of proinflammatory cytokine production including IL-23-induced IL-17 on activated CD4+ T cells partially through STAT3-dependent mechanism. J. Immunol. 177:5377-5385.

37. Lucas, S., Ghilardi, N., Li, J., and de Sauvage, F.J. 2003. IL-27 regulates IL-12 responsiveness of naive CD4+ T cells through Stat1-dependent and -independent mechanisms. Proc. Natl. Acad. Sci. U. S. A. 100:15047-15052.

38. Larousserie, F., et al. 2006. Differential effects of IL-27 on human B cell subsets. J. Immunol. 176:5890-5897.

39. Jouanguy, E., et al. 2007. Human primary immunodeficiencies of type I interferons. Biochimie. 89:878-883.

40. Casanova, J.L., and Abel, L. 2002. Genetic dissection of immunity to mycobacteria: The human model. Annu. Rev. Immunol. 20:581-620.

41. Notarangelo, L., et al. 2006. Primary immunodeficiency diseases: an update from the International Union of Immunological Societies Primary Immunodeficiency Diseases Classification Committee Meeting in Budapest, 2005. J. Allergy Clin. Immunol. 117:883-896.

42. Ochs, H.D., Smith, C.I.E., and Puck, J. 2006. Primary immunodeficiency diseases: a molecular and genetic approach. Oxford University Press. New York, New York, USA. 776 pp.

43. Minegishi, Y., et al. 2007. Dominant-negative mutations in the DNA-binding domain of STAT3 cause hyper-IgE syndrome. Nature. 448:1058-1062.

44. Caputi, M., Freund, M., Kammler, S., Asang, C., and Schaal, H. 2004. A bidirectional SF2/ ASF- and SRp40-dependent splicing enhancer regulates human immunodeficiency virus type $1 \mathrm{rev}, \mathrm{env}, \mathrm{vpu}$, and nef gene expression. J. Virol. 78:6517-6526.

45. Chen, H.H., Wang, Y.C., and Fann, M.J. 2006. Identification and characterization of the CDK12/ cyclin L1 complex involved in alternative splicing regulation. Mol. Cell. Biol. 26:2736-2745.

46. Sheppard, P., et al. 2003. IL-28, IL-29 and their class II cytokine receptor IL-28R. Nat. Immunol. 4:63-68.

47. Yoshida, H., et al. 2001. WSX-1 is required for the initiation of Th1 responses and resistance to $\mathrm{L}$. major infection. Immunity. 15:569-578.

48. Chen, Q., et al. 2000. Development of Th1-type immune responses requires the type I cytokine receptor TCCR. Nature. 407:916-920.

49. Schuetze, N., et al. 2005. IL-12 family members: differential kinetics of their TLR4-mediated induction by Salmonella enteritidis and the impact of IL-10 in bone marrow-derived macrophages. Int. Immunol. 17:649-659.

50. Cooper, A.M., Solache, A., and Khader, S.A. 2007. Interleukin-12 and tuberculosis: an old story revisited. Curr. Opin. Immunol. 19:441-447.

51. Kong, X.F., et al. 2007. MxA induction may predict sustained virologic responses of chronic hepatitis $B$ patients with IFN-alpha treatment. J. Interferon Cytokine Res. 27:809-818. 